\title{
Modeling the strain localization around an underground gallery with a hydro-mechanical double scale model; effect of anisotropy
}

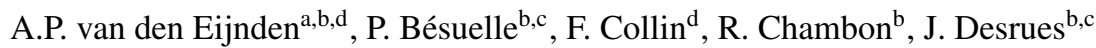 \\ ${ }^{a}$ Andra, 1-7 rue Jean Monnet, 92298 Châtenay-Malabry, France \\ ${ }^{b}$ Univ. Grenoble Alpes, 3SR, 38000 Grenoble, France \\ ${ }^{c}$ CNRS, 3SR, 38000 Grenoble, France \\ ${ }^{d}$ ArGEnCo dept, Univ. of Liège, 4000 Liège, Belgium
}

\begin{abstract}
This paper concerns the application of a finite element squared $\left(\mathrm{FE}^{2}\right)$ approach for modelling hydromechanical coupling in the simulation of gallery excavation in the context of radioactive waste repositories. The micromechanics of Callovo-Oxfordian claystone is modelled at the microscale through representative elementary volumes (REVs), taking into account the interaction of different mechanical constituents and its interaction with pore fluid. In a framework of computational homogenization, the micromechanical behaviour is coupled to the macroscale boundary value problem of a poromechanical continuum with local second gradient paradigm. The simulations concern several cases of the "Transverse action" benchmark by Andra, in the context of which the $\mathrm{FE}^{2}$ model is used. The explicit modelling of the material microstructure is used to introduce material anisotropy. The effect of the anisotropy in the initiation and evolution of strain localization in the excavation damaged zone (EDZ) around the gallery is studied and the influence on gallery convergence is demonstrated. A first-version model for solid-fluid interaction in the microstructure is applied here. As such, the objective of this work is to explore the capabilities of the model in capturing the general behaviour of the excavation damaged zone, rather than a quantitative reproduction of in-situ measurements. The application to the simulation of gallery excavation in the setting of the benchmark demonstrates the possibility of using the multiscale $\mathrm{FE}^{2}$ approach for assessing engineering problems.
\end{abstract}

Keywords: double scale model, computational homogenization, $\mathrm{FE}^{2}$, strain localization, gallery excavation, hydromechanical coupling

\section{Introduction}

In a recent paper (van den Eijnden et al., 2016), the framework of computational homogenization was extended to hydromechanical coupling and used in combination with a model for microstructural solid-fluid interaction to numerically derive the constitutive relations for a macroscale poromechanical boundary value problem. The application of the framework of computational homogenization provides a computationally efficient scale transition and has allowed the application of the $\mathrm{FE}^{2}$ method for hydromechanical coupling on (semi-) engineering problems. This paper presents the application of the $\mathrm{FE}^{2}$ model for hydromechanical coupling on the simulation of gallery excavations as part of the "Transverse action" benchmark by Andra (Seyedi et al., 2016).

The developments of the doublescale model in van den Eijnden et al. (2016) are based on a first-version microscale model for solid-fluid interaction at the grain scale. This microscale model, based on the work of Frey et al. (2012), captures some basic physical processes to represent degradation of geomaterials. No further conceptual improvements are made with respect to this model and therefore certain limitations with respect to capturing physical phenomena can be expected. The objective of this paper is to explore the capabilities qualitative performance of the doublescale framework with the first-version microscale model in capturing characteristic behaviour of an excavation damage zone (EDZ). Without conceptual modifications of the model, it is by no means the objective of this paper to quantitatively reproduce the in-situ measurements related to the benchmark project and for this reason, no quantitative comparison is made with in-situ measurements. Instead, the results are used to evaluate the possibilities and restrictions of the model and evaluate the effects of anisotropy introduced by the microstructure. 


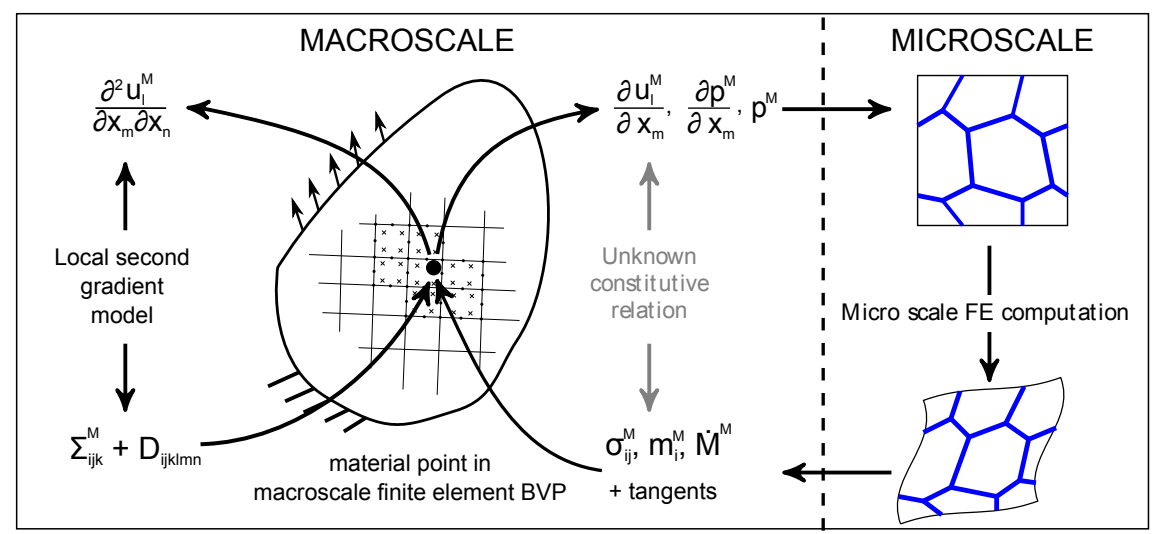

Figure 1: Schematic representation of the $\mathrm{FE}^{2}$ method for hydromechanical coupling with a local second gradient paradigm.

The double scale model is introduced in Section 2 with a description of the macro and micro field equations, the framework of scale transition and description of the algorithm for generating microstructure models. Section 3 gives a calibration example of the microscale model against experimental data. The boundary value problem of the 'Transverse action' benchmark is presented in Section 4 and results of different dry and wet cases are resumed in Sections 5 and 6, respectively. A discussion on the performance of the model close the paper.

\section{Double scale model with fully saturated hydro-mechanical coupling}

The employed multiscale modelling framework is the so-called finite element squared $\left(\mathrm{FE}^{2}\right)$ method (Terada and Kikuchi, 1995; Feyel and Chaboche, 2000). In this method the constitutive behaviour of each individual integration point in the macroscale finite element computation is derived from a boundary value problem (BVP) on a representative elementary volume (REV) at the microscale. The boundary conditions of this microscale BVP are dictated by the local kinematics of the macroscale problem. The homogenized response of the REV to the enforced global kinematics serves as a numerical constitutive relation at the macroscale. For deriving this homogenized response and its consistent linearization around the updated state without relying on numerical finite difference approximations which tend to be expensive, the framework of computational homogenization by static condensation was developed first for mechanical computation (Kouznetsova et al., 2001) and later extended for several types of coupled problems (Özdemir et al., 2008; Geers et al., 2010). An extension for hydromechanical coupling was proposed recently (van den Eijnden et al., 2016). This extension allows deriving a hydromechanical coupled constitutive relation for a poromechanical continuum from a REV containing details of the material microstructure (see Figure 1). The REV contains a granular assembly of solid parts interacting with pore fluid that can percolate through the pore network formed by the granular microstructure. The macroscale poromechanical continuum is enriched by means of a local second gradient paradigm to obtain mesh objective results in case of softening behaviour.

\subsection{Macro-scale formulation of a poromechanical second gradient continuum}

On the macroscale, a poromechanical continuum with hydromechanical coupling in a saturated porous medium is defined. For assessing material softening and localization phenomena in a finite element method without losing the objectivity of solutions due to the well-know mesh-dependency effects, a local second gradient paradigm (Chambon et al., 2001; Collin et al., 2006) is used for regularization. For the mechanical balance equations, this leads to the introduction of the double stress $\boldsymbol{\Sigma}$ with components $\Sigma_{i j k}$ as a dual to the microkinematical gradient $\boldsymbol{v}$, which in the so-called local second gradient models is constrained to be identical to the second gradient of displacement. This constraint in the strongest form leads to the balance equation for any kinematically admissible variation of displacement $u_{i}^{\star}$ :

$$
\int_{\Omega}\left(\sigma_{i j} \frac{\partial u_{i}^{\star}}{\partial x_{j}}+\Sigma_{i j k} \frac{\partial^{2} u_{i}^{\star}}{\partial x_{j} \partial x_{k}}\right) d \Omega-\int_{\Gamma}\left(\bar{t}_{i}^{\star}+\bar{T}_{i} \frac{\partial u_{i}^{\star}}{\partial x_{j}}\right) d \Gamma=0
$$


with $\sigma$ the Cauchy stress and $\bar{t}$ and $\bar{T}$ the boundary traction related to the first and second gradient parts. To solve this equation in a finite element method, without relying on higher continuous elements, the constraint on $v$ is weakened by means of Lagrange multipliers, introducing fields of Lagrange multipliers as additional variables to solve for (Chambon et al., 2001; Matsushima et al., 2002). The balance equation for the fluid phase remains classical. For fluid mass flux $\vec{m}$ and fluid mass density $M$ the balance equation for any kinematically admissible variation of pore pressure $p^{\star}$ over an arbitrary domain $\Omega$ is written as

$$
\int_{\Omega}\left(m_{j} \frac{\partial p^{\star}}{\partial x_{j}}-\dot{M} p^{\star}\right) d \Omega-\int_{\Gamma} \bar{q} p^{\star} d \Gamma=0,
$$

where $\bar{q}$ is the fluid mass flux over domain boundary $\Gamma . \dot{M}$ is the time derivative of fluid mass $M$. Finite element discretization allows solving these nonlinear field equations for prescribed boundary conditions in an iterative way. The algorithm was implemented in the finite element code Lagamine (Charlier, 1987; Collin et al., 2006). The local second gradient paradigm provides the assumption of decoupling between the classical part of the model and the second gradient part, which is of vital importance to the coupling with the framework of computational homogenization; the first and second gradient part of the model can therefore be formulated independently. A general expression of the constitutive relations, with coupling between solid and fluid phases in the first gradient part, can therefore be formulated as

$$
\left[\begin{array}{ccc}
C_{i j k l} & A_{i j l} & B_{i j} \\
E_{i k l} & G_{i l} & H_{i} \\
K_{k l} & L_{l} & N
\end{array}\right]\left\{\begin{array}{l}
\frac{\partial \delta u_{k}}{\partial x_{l}} \\
\frac{\partial \delta p}{\partial x_{l}} \\
\delta p
\end{array}\right\}=\left\{\begin{array}{l}
\delta \sigma_{i j} \\
\delta m_{i} \\
\delta \dot{M}
\end{array}\right\}
$$

and

$$
D_{i j k l m n}^{S G} \frac{\partial \delta v_{l m}}{\partial x_{n}}=\delta \Sigma_{i j k}
$$

The latter is formulated as a phenomenological relation between increments of the microkinematical gradient tensor to increments of the double stress in the framework of micromorphic continuum (Germain, 1973), for which only an elastic relation (Mindlin, 1964) is considered here. When restricted to isotropy of the second gradient model, the sixth-order tensor $\boldsymbol{D}^{S G}$ can be expressed through a single parameter $D[N]$, implicitly controlling the width of strain localization bands (Chambon and Caillerie, 1999; Bésuelle et al., 2006). This parameter needs calibration against the constitutive relation and the mesh size in order to guarantee mesh-objective results in case of localization phenomena.

Equation (3) is the general expression of the classical part of the model, which is coupled to the micromechanical model in the framework of first order computational homogenization. The left hand matrix, containing the tangent operators, is derived from the microscale material response to a variation of the kinematics increments through computational homogenization (see van den Eijnden et al. (2016) for details). The consistency of these tangent operators is with respect to the current kinematics loading directions. Terms $C_{i j k l}$ form the stiffness matrix; terms $B_{i j}$ control the increase of total stress as a response to increase in pore pressure and can be compared with parameter $b$ in Biot theory (Biot, 1941); terms $E_{i k l}$ describe the dependency of the permeability on deformation; $G_{i l}$ contains the permeability tensor scaled by the fluid density; $H_{i}$ and $N$ hold information on the fluid compressibility and are scaled by fluid fluxes and fluid mass content respectively; terms $K_{k l}$ contains information on the evolution of pore volume through deformation; remaining terms are, as an effect of the microscale model applied here, equal to zero. A more detailed evaluation of the different components of the tangent operators, and their physical interpretation in terms of poromechanical constitutive relations, can be found in Marinelli et al. (2016).

\subsection{Scale transition in a computational homogenization framework}

Local periodicity of the microstructure is assumed, providing a straightforward formulation of the REV boundary conditions for microscale degrees of freedom $u_{i}^{m}$ (displacement) and $p^{m}$ (fluid pressure) in relation with the local macroscale kinematics $u_{i}^{M}, p^{M}$ :

$$
\begin{aligned}
& \vec{u}^{m}\left(\vec{x}^{+}\right)-\vec{u}^{m}(\vec{x})=\nabla \vec{u}^{M} \cdot\left(\vec{x}^{+}-\vec{x}\right) \\
& \hat{p}^{m}\left(\vec{x}^{+}\right)-\hat{p}^{m}(\vec{x})=\nabla p^{M} \cdot\left(\vec{x}^{+}-\vec{x}^{-}\right)
\end{aligned}
$$


with $\vec{x}^{+}$and $\vec{x}^{-}$the homologous couples of coordinates on the periodic REV boundary $\Gamma$ and $\hat{p}$ the microscopic fluctuation of the microscale pore pressure $p^{m}$ according to

$$
p^{m}=p^{M}+\hat{p}, \quad p^{M} \approx p^{m}
$$

The average pressure over the REV is prescribed by the local macroscale pressure $p^{M}$ :

$$
p^{M}=\frac{1}{\Omega_{R E V}^{h}} \int_{\Omega_{R E V}^{h}} p^{m} d V
$$

Domain $\Omega^{h}$ indicates the part of the total domain occupied by the fluid phase, as explicitly defined in the microscale model.

After solving the REV boundary value problem by means of the finite element method, the homogenized macroscale response of total stress $\sigma_{i j}^{M}$, fluid mass flux $m_{i}^{M}$, and the fluid mass storage $\dot{M}$ is derived from the equilibrated REV based on Hills condition for macro homogeneity. This condition prescribes equal (virtual) work at both scales. From this relation, the homogenized response of the REV to prescribed macroscale kinematics is derived (van den Eijnden et al., 2016):

$$
\begin{gathered}
\sigma_{i j}^{M}=\frac{1}{\Omega_{R E V}} \int_{\Gamma^{+}} \bar{t}_{i}^{+}\left(x_{j}^{+}-x_{j}^{-}\right) d \Gamma \\
m_{i}^{M}=\frac{1}{\Omega_{R E V}} \int_{\Gamma^{+}} \bar{q}^{+}\left(x_{i}^{+}-x_{i}^{-}\right) d \Gamma \\
\dot{M}=\frac{M^{t}-M^{t-\Delta t}}{\Delta t}, \quad M^{t}=\frac{1}{\Omega_{R E V}} \int_{\Omega_{R E V}^{h}} \rho^{w} d \Omega
\end{gathered}
$$

with $\bar{t}_{i}^{+}$the REV boundary traction and $\bar{q}^{+}$the boundary flux on REV boundary $\Gamma^{+}$. These terms are antiperiodic with respect to $\bar{t}_{i}^{-}$and $\bar{q}^{-}$on boundary $\Gamma^{-}$, the homologous counterpart of $\Gamma^{+} . M^{t}$ is fluid mass per unit volume at time $t$. Note that in the expressions given above, divergence theorem and the anti-periodic boundary response was applied to transform volume integrals into boundary integrals. This expression can be derived from a volume integral over the (piecewise continuous) microstructure (van den Eijnden et al., 2016).

For deriving the consistent linearization of the homogenized response around the updated configuration, computational homogenization is employed to condense the microscale global systems of equations used to solve the microscale BVP on the variations of macroscale kinematics $\left\{\nabla \delta u^{M}, \nabla \delta p^{M}, \delta p^{M}\right\}$ and macroscale response $\{\delta \sigma, \delta \vec{m}, \delta \dot{M}\}$. The resulting linearization is consistent with the kinematics of the updated configuration and provides the necessary consistent tangent operators of (3) in the Newton-Raphson iterative scheme for solving the macroscale BVP. The development of the computational homogenization for deriving the tangent operators lies outside the scope of this work as it is only relevant to the efficiency of the numerical scheme for solving the macroscale BVP. The reader is referred to van den Eijnden et al. (2016) for a detailed description of its development.

\subsection{Micro-scale model for hydromechanical solid-fluid interaction}

At the microscale, the material microstructure is modelled as a granular assembly of solid or porous units (grains) separated by cohesive interfaces, using a model based on Frey et al. (2012). These interfaces form a network of pore channels between the grains through which fluid can percolate (Figure 2). Hydromechanical coupling is thereby modelled as the interaction of micromechanical solid and fluid phases rather than by a phenomenological description. The solid phases form a granular microstructure, in which individual grains represent different components of the material microstructure. Grains are considered linear elastic and separated by cohesive interfaces. Normal and tangential cohesive forces are defined independently, using a simplistic damage law depending on parameters $T_{t / n}^{\max }$ (the maximum cohesive force tangential or normal to the grain boundary), $0<D_{t / n}^{t} \leq 1$ (the relative degradation of the interface) and 


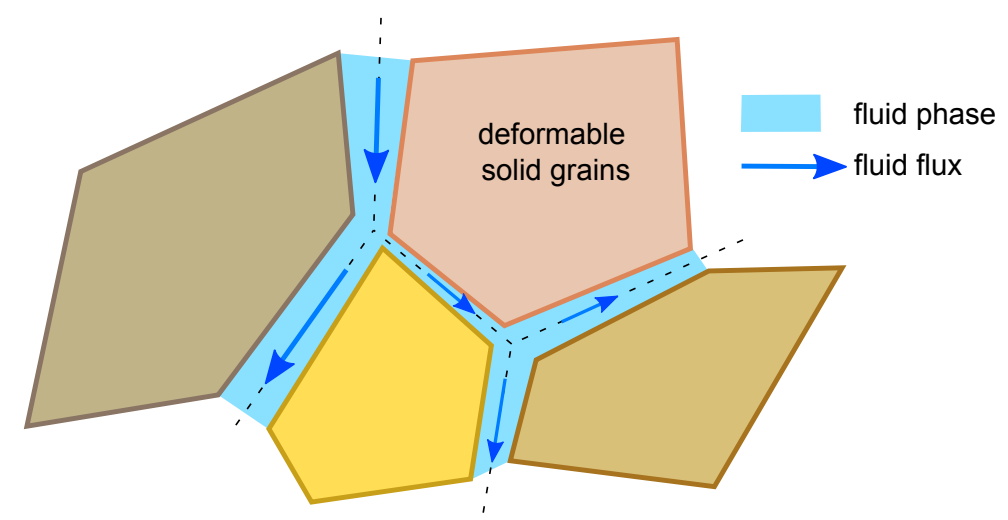

Figure 2: Concept of the micromechanical model
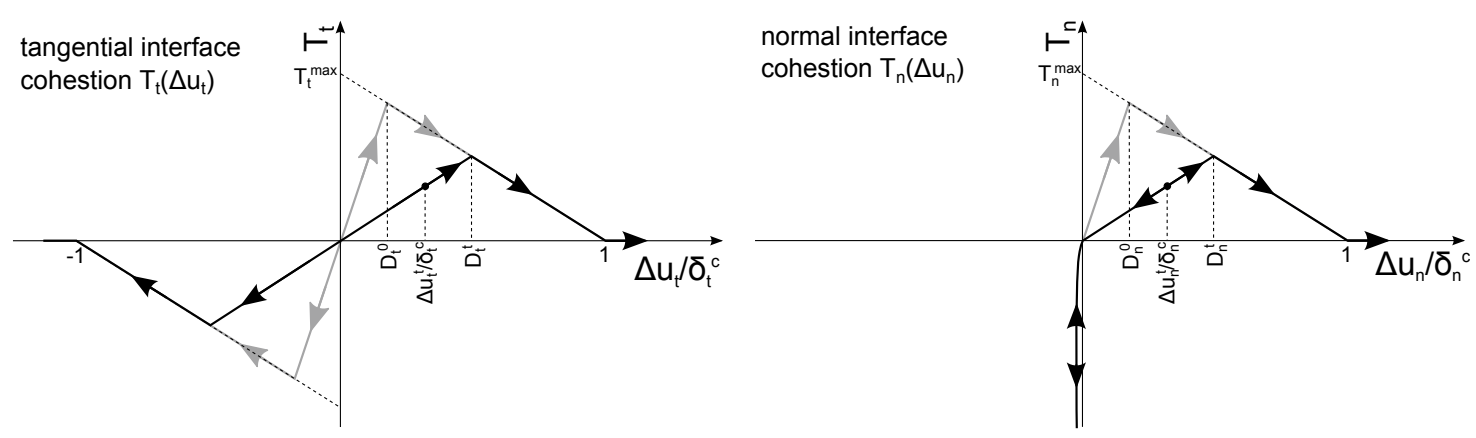

Figure 3: Linear damage model for interface cohesion components $T_{t}$ and $T_{n}$.

$\delta_{t / n}^{c}$ (the relative interface displacement for complete degradation of the cohesive forces). Interface state parameters $D_{t / n}^{t}$ take into account the history of the relative displacement $\Delta u_{t / n}$ between the opposite sides of the interface:

$$
\begin{aligned}
D_{t}^{t} & =\max _{\tau=0 \ldots t}\left(D_{t}^{0},\left|\Delta u_{t}^{\tau}\right| / \delta_{t}^{c}\right) \\
D_{n}^{t} & =\max _{\tau=0 \ldots . t}\left(D_{n}^{0}, \Delta u_{n}^{\tau} / \delta_{n}^{c}\right)
\end{aligned}
$$

where $D_{t / n}^{0}$ are model parameters defining the state of initial degradation and thereby the initial interface stiffness. The state variables $D_{t / n}^{t}$ allow writing the equations for the interface cohesion (see also Figure 3):

$$
\begin{aligned}
T_{t}^{t} & =T_{t}^{\max }\left(1-D_{t}^{t}\right) \frac{\Delta u_{t}^{t}}{D_{t}^{t} \delta_{t}^{c}} & & \\
T_{n}^{t} & =T_{n}^{\max }\left(1-D_{n}^{t}\right) \frac{\Delta u_{n}^{t}}{D_{n}^{t} \delta_{n}^{c}} & & \text { if } \Delta u_{n}^{t}>=0 \\
& =T_{n}^{\max }\left(1-D_{n}^{t}\right) \frac{\Delta u_{n}^{t}}{D_{n}^{t} \delta_{n}^{c}}-\chi \Delta u_{n}^{t^{2}} & & \text { if } \Delta u_{n}^{t}<0
\end{aligned}
$$

The additional term $-\chi \Delta u_{n}^{t^{2}}$ is used to take into account normal contact of grains by means of penalization with $\chi>>T_{n}^{\max }$.

As a result of the separation of scales, the microscale problems can be solved in steady state conditions and fluid storage is only a macroscale phenomenon. Fluid compressibility is taken into account as

$$
\rho^{w}=\rho^{0} \exp \left(\frac{p^{m}}{k^{w}}\right)
$$




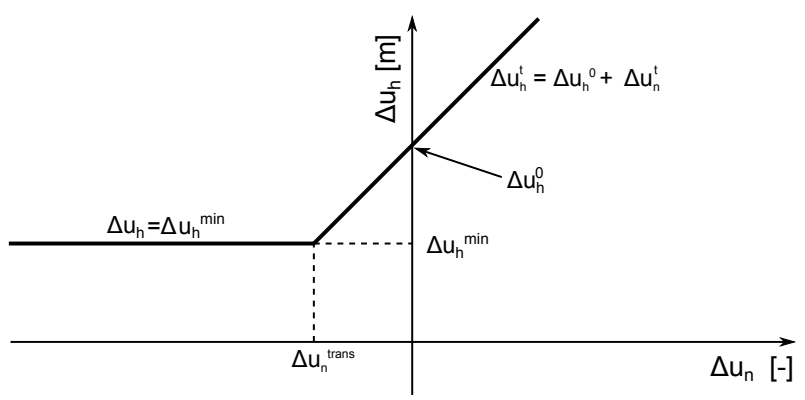

Figure 4: Relation between normal interface opening $\Delta u_{n}$ and hydraulic equivalent opening $\Delta u_{h}[l]$

with $k^{w}$ the fluid bulk modulus and $\rho^{0}$ the fluid density at zero pressure.

Following the separation of scales, the spatial variation of fluid pressure within the REV can be neglected with respect to the average pressure (Equation (7)). This means that for a given macroscale fluid pressure all microscale fluid pressures $p^{m}(\vec{x})$ are known before solving the microscale balance equations. Therefore the (nonlinear) mechanical microscale problem can be solved for a given macroscale deformation gradient, independent from the hydraulic microscale problem. Once the mechanical microscale problem is solved and the aperture of interfaces $\Delta u_{n}$ is known, the hydraulic problem can be solved.

Poiseuille flow between parallel plates at both sides of the equivalent hydraulic interface opening $\Delta u_{h}=f\left(\Delta u_{n}\right)$, is used to model fluid flow in the interface channels. The relation between $\Delta u_{h}$ (used for in the hydraulic problem) and $\Delta u_{n}$ (the relative displacement normal to the interface) plays an important role in the hydromechanical coupling, as it directly controls the hydraulic conductivity of the interfaces. The relation is graphically presented in Figure 4. A minimum hydraulic opening guarantees that non-physical negative hydraulic conductivity is avoided and allows enforcing an initial and a minimum homogenized permeability. Note moreover that $\Delta u_{h}$ has a length scale relative to the fluid viscosity. It thereby implicitly introduces a microstructural length scale as soon as permeability is considered. In this work a ratio $\partial \Delta u_{h} / \partial \Delta u_{n}=1 \mathrm{~mm}$ is used, leading indirectly to an REV of dimensions $1 \mathrm{~mm} \times 1 \mathrm{~mm}$. Parameters $\Delta u_{h}^{\min }$ and $\Delta u_{n}^{\text {trans }}$ are used as model parameters to calibrate the intrinsic permeability of the material.

At location $s$ in the interface, the fluid mass flux per unit thickness $\varpi$ flowing through the interface is found as

$$
\varpi(s)=-\rho^{w} \frac{\Delta u_{h}(s)^{3}}{12 \mu} \frac{d \hat{p}}{d s}=-\rho^{w} \kappa(s) \frac{d \hat{p}}{d s}
$$

with $\mu$ the fluid viscosity. Considering fluid mass conservation in the channel as result of the steady state conditions in the REV, (17) can be integrated over channel $l$ and an expression for the fluid mass flux as a function of nodal pressure at the beginning $\left(\hat{p}^{k-}\right)$ and end $\left(\hat{p}^{k+}\right)$ of the channel is found:

$$
\varpi^{l}=\phi^{l}\left(\hat{p}^{l+}-\hat{p}^{l-}\right)
$$

The conductivity term contains the integral of $k(s)$ over the interface channel:

$$
\phi^{k}=\rho^{w}\left(\int_{s^{k-}}^{s^{k+}} \frac{1}{\kappa(s)} d s\right)^{-1}
$$

Integration is performed by Gauss quadrature over interface elements. Mass balance equations $q^{m}=\sum \varpi=0$ can be solved for the nodes of the interface elements. Global assembly of (18) gives the global system of equations for the fluid transport:

$$
\left[K^{h h}\right]\left\{\hat{p}^{m}\right\}=\left\{q^{m}\right\}
$$

This system of equations can be solved directly by enforcing the periodic boundary conditions (6), after which residual nodal fluxes at the periodic boundary $\Gamma^{+}$are used to numerically solve the boundary integral of the fluid mass flux in (10). 


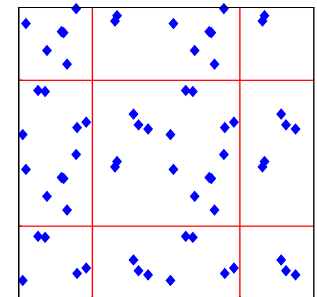

a) vectors $z$

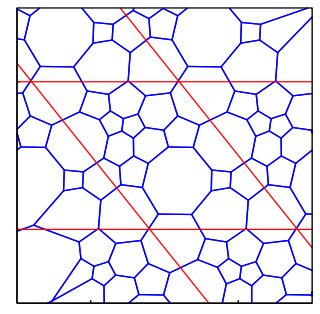

d) shape correction

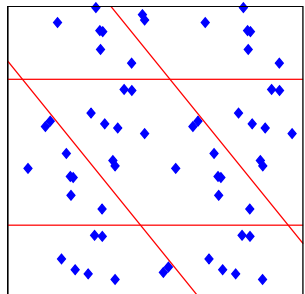

b) transformation $\mathbf{T}^{-1}$

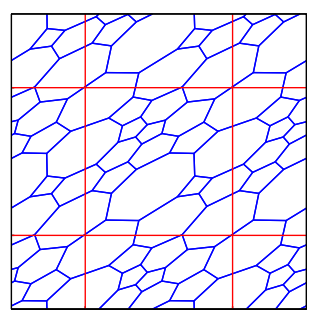

e) reverse transformation

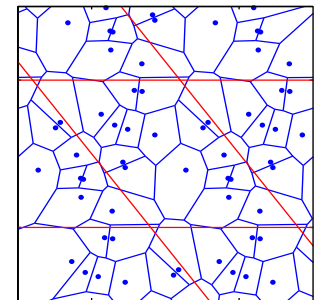

c) Voronoi tessellation

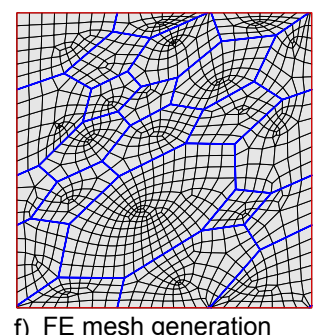

Figure 5: Illustration of the algorithm for anisotropic REV generation with grain shape tensor $T=\left[\begin{array}{llll}1 & 0.8 ; & 0 & 1\end{array}\right]$ and correction parameter $\eta=1$. Red boundaries indicate the periodic domain $\Omega$ (van den Eijnden (2015))

\subsection{Algorithm for generating microstructures}

An algorithm based on Voronoï tessellation is adopted to generate periodic REVs with random microstructures. Several control parameters are added to the tessellation algorithm to be able to control the grain geometry statistics. These parameters can be used to accurately match the geometrical characteristics of the geometry of inclusions of the claystone as characterized by Robinet et al. (2012). In this work, the control parameters are used in an attempt to control the strength anisotropy. These parameters are introduced in the algorithm for generating microstructural REVs as presented in Figure 5:

a) A set of $n_{\text {grain }}$ sites $\vec{z}$ is generated in a periodically repeated domain $\Omega$.

b) Domain $\Omega$ is then transformed by shape tensor $\boldsymbol{T}$ into $\Omega^{T}$ through $\vec{z}^{T}=\boldsymbol{T}^{-1} \vec{z}$ with $\boldsymbol{T}$ defined as

$$
\boldsymbol{T}=\left[\begin{array}{cc}
\xi \cos (\beta) & \sin (\beta) \\
-\xi \sin (\beta) & \cos (\beta)
\end{array}\right]
$$

parameter $\xi$ defines the anisotropy stretch and parameter $\beta$ a bedding orientation

c) Voronoï tessellation is applied on sites $z^{T}$ in $\Omega^{T}$ to find the Voronoï diagram that forms the basis of the microstructure, defined by the connectivity between Voronoï vertices $\vec{x}_{A}^{T}$.

d) A shape correction based on minimization of the sum of the vertex connection lengths squared, is applied to avoid grains with a high angularity;

$$
\frac{\partial L}{\partial x_{i_{I}}^{T}}=0, \quad L=\sum_{I=1}^{m} \sum_{J=1}^{n c_{I}}\left|\vec{x}_{I}^{T}-\vec{x}_{I, J}^{T}\right|^{2}
$$

where $\vec{x}_{I, J}^{T}$ is one of the $n c_{I}$ vertices directly connected to vertex $\vec{x}_{I}^{T}$, resulting in $\vec{x}_{B}^{T}$

e) A fraction $0 \leq \eta \leq 1$ of the shape correction is then taken into account as a linear combination of $\vec{x}_{B}^{T}$ and $\vec{x}_{A}^{T}$ as

$$
\vec{x}^{T}=\vec{x}_{A}^{T}(1-\eta)+\vec{x}_{B}^{T} \eta
$$

f) The transformed microstructure $\vec{x}^{T}$ is transformed back through the operation $\vec{x}=\boldsymbol{T}^{-1} \vec{x}^{T}$ after which the microstructure is discretized by means of a finite element mesh and material properties are assigned to the different components of the microstructure. 

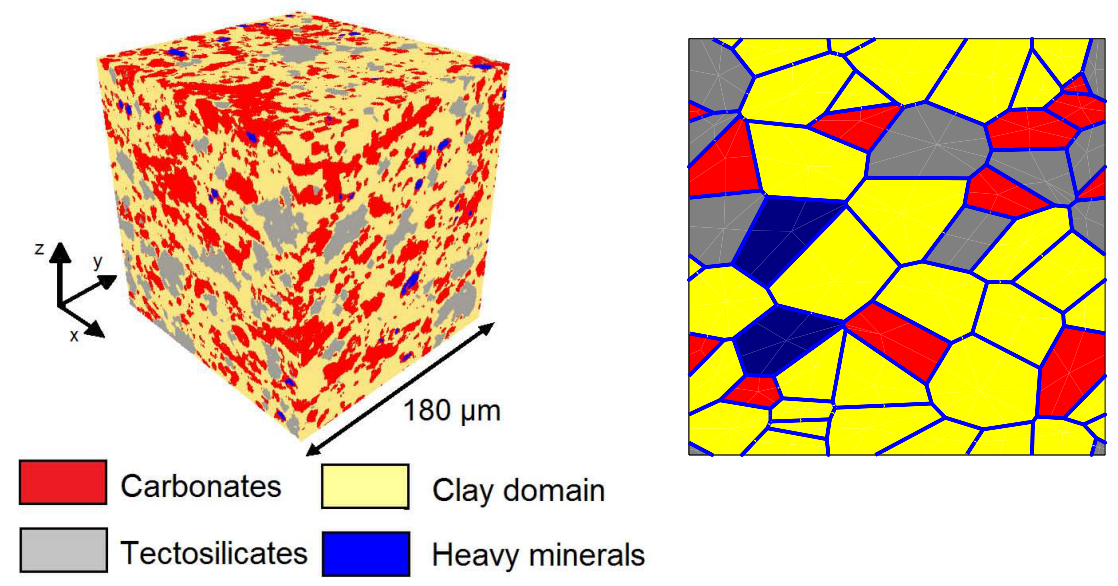

Figure 6: Left: a discretization of an X-ray tomography image of the COx microstructure (Robinet et al., 2012). Right: a model realization with equal volume fractions as generated by Voronoi tessellation.

\begin{tabular}{l|ccccccc|cc} 
solids: & $-\mathrm{Si}_{x} \mathrm{O}_{y}$ & $-\mathrm{CO}_{3}$ & $\mathrm{FeS}_{2}$ & clay & & interfaces: & & geometry: \\
\hline Volume fraction* & 0.13 & 0.25 & 0.03 & 0.60 & & $\delta_{t / n}^{c}$ & 0.05 & $\beta^{\text {bed }}$ & $10^{\circ}$ \\
$E$ [GPa] & 95 & 84 & 305 & 2.3 & & $D_{t / n}^{0}$ & 0.002 & $\eta$ & 0.1 \\
$v[-]$ & 0.074 & 0.317 & 0.154 & 0.11 & & $T_{n}^{\text {max }}[\mathrm{MPa}]$ & 1.0 & $\chi$ & 1.3 \\
& & & & & & $T_{t}^{\text {max }}[\mathrm{MPa}]$ & 5.5 & &
\end{tabular}

Table 1: Micromechanical properties for the microstructural model in Figure 6. * After Robinet et al. (2012)

\section{Modelling local material behaviour}

In the doublescale model the local macroscale material behaviour is derived from the REV boundary value problem on the microstructure. This means that the local behaviour of the material can be studied by means of a single REV, on which a controlled loading path (stress, strain or mixed) is enforced. Local material behaviour, characterized by different microstructures, is studied here and calibrated against experimental data.

\subsection{Microstructure calibration example}

The microscale model aims at capturing the behaviour of the claystone at the level of the inclusions in the range of $20-100 \mu \mathrm{m}$ as observed for example in Robinet et al. (2012) (see Figure 6). Inclusions consist of carbonates, quartz and calcite, embedded in a clay matrix (Andra, 2005). Since the model does not provide the means for taking into account a continuous clay matrix, possible cracks are assumed, represented by the Voronoi diagrams. Grains are selected randomly to assign material properties of inclusions and clay matrix according to the volume fractions observed in the claystone (see Table 1). This means that the different types of inclusions have equal distributions of shapes. Comparing geometric distributions (elongation and orientation) between inclusions in the claystone and Voronoi diagrams, grain shape parameters can be calibrated as bedding $\beta^{\text {bed }}=10^{\circ}$, shape correction factor $\eta=0.1$ and elongation $\chi=1.3$. Comparison of the distribution of grain elongation and orientation with measurements performed by Robinet et al. (2012) show very good agreement between basic geometrical characteristics of the Voronoi-based model and the inclusions of COx claystone (van den Eijnden, 2015). Other geometric characterization variables (asperity for example) were not considered for comparison.

For selecting the material parameters, the elastic properties of calcite (carbonates), quartz (tectosilicates) and pyrite (heavy minerals) are used. As the elastic properties of the clay matrix are unknown, they are calibrated using the elastic properties of the homogenised response in comparison with laboratory tests. The resulting elasticity parameters are given in Table 1. The interface properties are then calibrated against triaxial test data at confining stress levels of $2 \mathrm{MPa}$ and $12 \mathrm{MPa}$ as provided for the benchmark (Andra, 2013). 

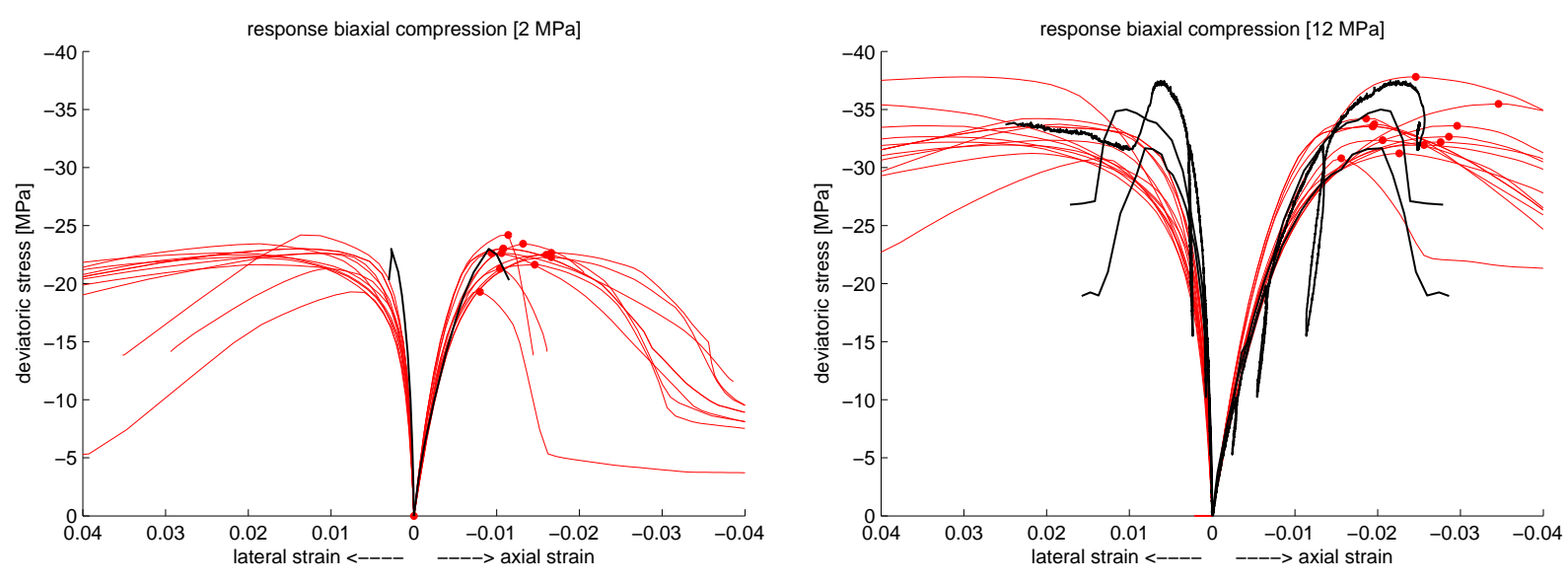

Figure 7: Deviatoric stress response to biaxial material point compression for orientations at an interval of $15^{\circ}$. In black the experimental results, obtained by triaxial compression.

The results of 4 experimental triaxial compression tests (one at $2 \mathrm{MPa}$ confinement, three at $12 \mathrm{MPa}$ confinement (Andra, 2013)) are used for the calibration. Their stress-strain curves are given in Figure 7 together with the results of the calibrated numerical response to biaxial compression for different REV orientations. They show a good agreement in initial stiffness, peak stress, confining stress dependency and axial strain at peak stress. Lateral strain shows good agreement at low stress levels, but the numerical results show a stronger dilatancy at higher stress levels and close to the point where softening takes place. This high degree of dilatancy is strongly linked to the fact that relative displacement between grains (which is needed for interface softening) can only be obtained by the opening of adjacent interfaces. As rearrangement of grains can not be taken into account in the finite element formulation, this relative opening of the interfaces continues regardless of the state of deformation. Moreover, the modelled response to biaxial compression is that of a material point, whereas the experimental results are nominal responses of larger material samples and can not be considered to represent local behaviour as soon as non-homogeneous modes of deformation takes place, possibly well before reaching peak strength .

From a mechanical point of view, microstructure dimensions are all relative to the REV. As a result, interface cohesion laws are relative to the grainsize, which is dimensionless. In case of hydromechanical coupling, a length scale is required and the size of the REV is implicitly introduced in the hydraulic coupling as discussed in Section 2.3.

The results in this section demonstrate that with sufficient grains taken into account in the REV, the local mechanical behaviour of the COx claystone can be accurately reproduced. Nevertheless, the model remains two-dimensional and only plane strain conditions can be taken into account.

\subsection{Selected REVs for micro-scale model}

In the following, a simpler set of REVs is used for modelling the microstructure in doublescale computations. Several series of random realizations of microstructures were generated with different geometry parameters. From these series a selection was made based on the anisotropy in peak response to biaxial compression. Because a low number of grains is considered, grain stiffness was chosen isotropic and homogeneous over the REV with $E=4.0 \mathrm{GPa}$ and $v=0.2$. Interfaces are initially rigid with respect to the grain stiffness, such that the homogenized stiffness of the microstructure initially is dominated by the stiffness of the grains. For simplicity, interface cohesion parameters were chosen homogeneous over the REV. Four microstructures were chosen for the simulation, as presented in Figure 8. Two of them have a low anisotropy of peak strength (micro_2 and micro_28), and the two others a stronger anisotropy (micro_11 and micro_16) (Figure 9). As experimental data of micromechanical constitutive parameters are not available, the interface cohesion parameters are calibrated such that the homogenized response of the REVs corresponds to laboratory triaxial compression tests assumed to represent the local material behaviour. Experimental triaxial test results are compared with numerical biaxial test results (plane strain). As the main purpose of this calibration is the qualitative comparison of the different REVs, the differences between triaxial and biaxial compression as a possible 


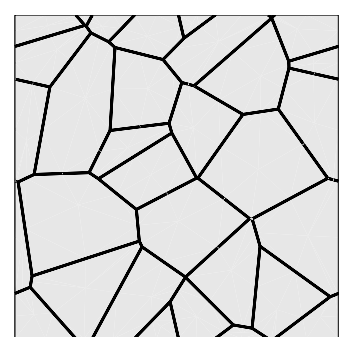

micro_2

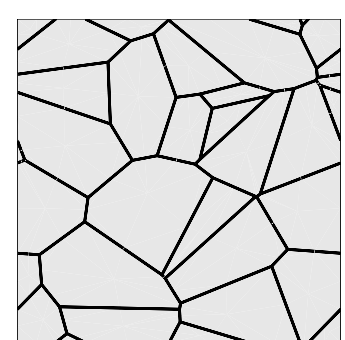

micro_11

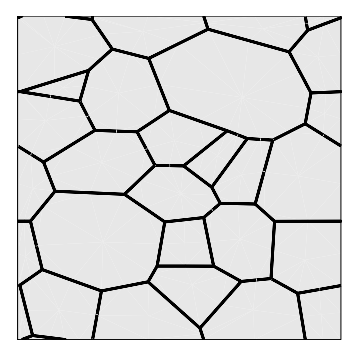

micro_16

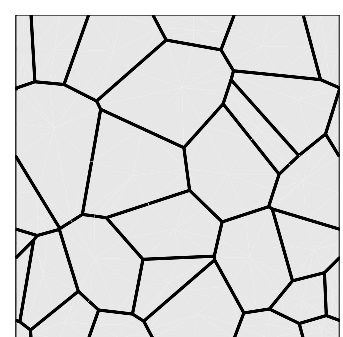

micro_28

Figure 8: Selected microstructures for the doublescale simulations.

\begin{tabular}{l|cccccc|cccc} 
REV & $\eta$ & $\xi$ & $\beta$ & $\theta^{R E V}$ & $n$ & & & $T_{t / n}^{\max }$ & $\delta^{c}$ & $D^{0}$ \\
\hline micro_2 & 0.1 & 1.00 & $0^{\circ}$ & $60^{\circ}$ & 20 & & micro_2 & $4.0 / 2.0$ & 0.080 & 0.001 \\
micro_11 & 0.1 & 1.28 & $0^{\circ}$ & $0^{\circ}$ & 20 & & micro_11 & $4.0 / 0.1$ & 0.100 & 0.002 \\
micro_16 & 0.5 & 1.28 & $0^{\circ}$ & $0^{\circ}$ & 20 & & micro_16 & $3.0 / 0.1$ & 0.100 & 0.002 \\
micro_28 & 0.2 & 1.00 & $0^{\circ}$ & $-80^{\circ}$ & 20 & & micro_28 & $1.0 / 1.0$ & 0.125 & 0.010
\end{tabular}

Table 2: Microstructure properties: circularity correction parameter $\eta$, stretch factor $\xi$, bedding plane $\beta$, REV rotation $\theta^{R E V}$ and number of grains $n$

effect of different Lode angles in both strength and stiffness are ignored. Calibration is done by means of first the grain stiffness and later interface cohesive parameters $T_{t / n}^{\max }$ and $\delta_{t / n}^{c}$. The grain stiffness is used to calibrate the initial stiffness of the global response, the cohesion parameters are used for calibrating the peak strength and peak strength deformation. Figure 10 shows the result of calibration at 2.0 $\mathrm{MPa}$ and $12.0 \mathrm{MPa}$ lateral stress with a comparison between experimental results (Andra, 2013; Armand et al., 2016) and homogenized material response. The calibrated interface cohesion parameters for the four REVs are given in Table 2. Comparison with experimental curves shows that calibrated microstructures can fit experimental data to a reasonable extend and reproduce well the mean stress dependency (micro_2), can underestimate the stress peak at low confining pressure and fit well at high confining pressure (micro_11 and micro_28), or can fit well at low confining pressure and overestimate stress peak at high confining pressure (micro_16). In absence of experimental data on stress peak anisotropy, it is difficult to go further in the comparison.

An anisotropic permeability tensor is an intrinsic part of the material behaviour derived from the microscale computations. The permeability of the model is controlled by the interface aperture. A correction of the aperture with respect to the equivalent hydraulic interface opening $\Delta u_{h}$ is introduced to prescribe a minimum opening of the interfaces in terms of fluid percolation (see Section 2.3). This allows to control the permeability of the homogenized response for undeformed microstructures. Although anisotropy of the permeability tensor is an inherent part of the model introduced by the distribution of interface channels, in undeformed microstructures the grains need to have a strong bedding orientation to result in any significant anisotropy of the permeability. As a result, the REVs presented in this work have practically isotropic permeability tensors in initial state as anisotropy is only introduced by means of microstructure geometry. Alternative ways of introducing stronger anisotropy in the homogenised response would be to select the minimum hydraulic opening of a channel as a function of its orientation (based on for example sedimentological arguments) or the incorporation of phenomenological description of fluid transport by diffusive flow through the grains (van den Eijnden, 2015). These types of enhancements would however introduce phenomenological descriptions in the microscale model, which contradicts the motivation of using a microscale model for solid-fluid interaction.

In this work, the hydraulic equivalent interface openings of undeformed material $\Delta u_{h}^{0}$ were defined homogeneous over the REV and equal to $0.05 \mu \mathrm{m}$. This leads to an initial permeability in the order of $5.0 E^{-20} \mathrm{~m}^{2}$.

\section{Solved initial boundary value problems}

The excavation of a gallery in the Meuse/Haute-Marne Underground Research Laboratory (URL) is simulated in two configurations with respect to the principal stress directions. The in situ principal stresses, at $490 \mathrm{~m}$ depth, are 

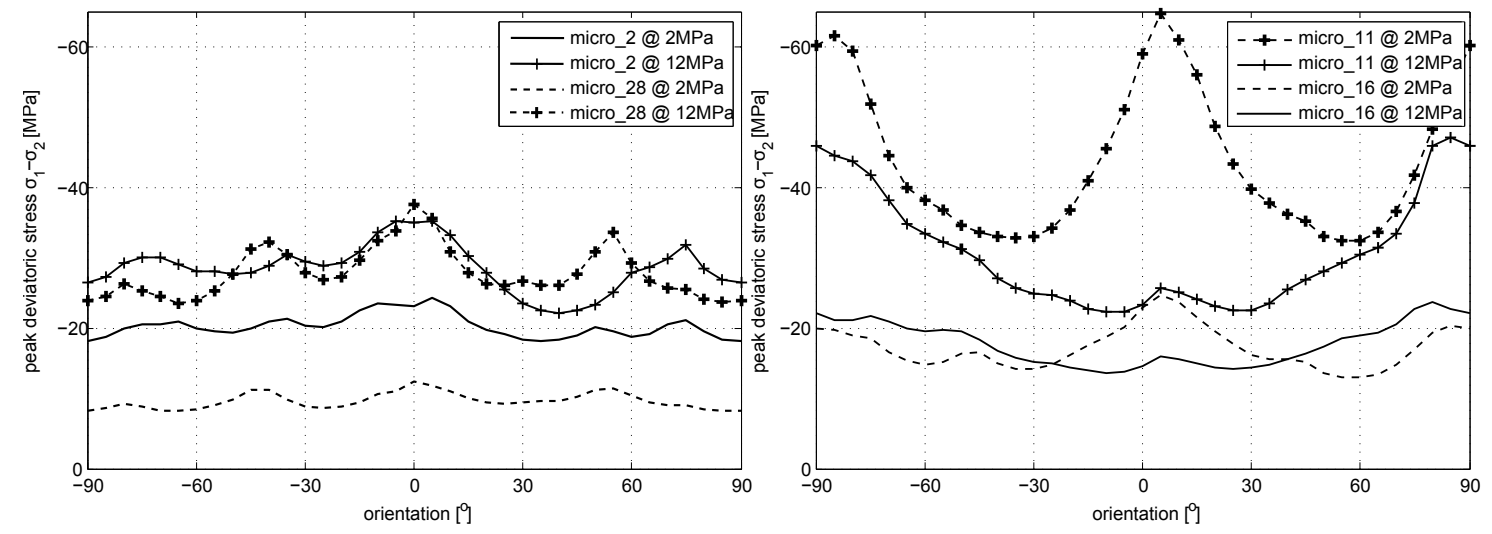

Figure 9: Anisotropy in the peak response to biaxial loading for the 4 microstructural REVs under $2 \mathrm{MPa}$ and $12 \mathrm{MPa}$ confining stress.

estimated to be $\sigma_{v}=12.7 \mathrm{MPa}$ in the vertical direction, $\sigma_{h}=12.4 \mathrm{MPa}$ in a horizontal direction, corresponding to the minor principal direction, and $\sigma_{H}=16.1 \mathrm{MPa}$ in the orthogonal horizontal direction, corresponding to the major principal stress (Seyedi et al., 2016). Figure 11 shows two cases of gallery orientation with respect to principal stress directions. In case $A$, the excavation is oriented in the direction of major principal stress $\left(\sigma_{H}\right)$. This means that the stress components in a plane orthogonal to the gallery axis can be considered isotropic since $\sigma_{h} \approx \sigma_{v}$. In case $\mathrm{B}$, the gallery axis is parallel to the minor principal stress direction $\left(\sigma_{h}\right)$. The upper right quartiles of the gallery cross-sections will be modelled.

The gallery radius is $2.6 \mathrm{~m}$. The considered rock mass is a thick quarter hollow cylinder with an external radius equal to $50 \mathrm{~m}$ (Figure 12). All computations are performed under plane strain hypothesis to model the behavior of a vertical section orthogonal to the gallery. A symmetry of the solution is assumed both on the horizontal bottom boundary and the vertical left boundary, by imposing, respectively, zero vertical displacement and zero horizontal displacement. These assumption are strong in presence of anisotropy and strain localization, as will be discussed later. For case A, the initial stress is considered isotropic, equal to $\sigma_{h}$. In case B, the initial stress is equal to $\sigma_{H}$ in the horizontal direction, and $\sigma_{v}$ in the vertical direction. Therefore, the boundary tractions related to the far-field (blue boundary) and the gallery wall (red boundary) depend on the position of the considered node, and have both a normal and a tangential component. These components are expressed as the product of the stress tensor and the unit normal outward vector. Constant traction is applied on the external boundary, while a time dependent traction is applied on the internal boundary, using the linear relation

$$
\vec{\tau}_{\text {gallery }}=\left(\vec{\tau}_{0}-\vec{\tau}_{\text {shield }}\right)(1-\lambda)+\vec{\tau}_{\text {shield }},
$$

where $\vec{\tau}_{\text {shield }}$ represents the residual traction that should sustain the internal shield after convergence of the gallery and $\lambda$ a loading parameter to control the unloading of the gallery wall. The traction $\vec{\tau}_{\text {shield }}$ is considered to act in a direction normal to the gallery wall at $0.3 \mathrm{MPa}$, as a given boundary condition of the benchmark.

For a first series of computations, the fluid is not taken into account in the model. The aim is to evaluate the ability of the double scale model to predict failure during the gallery excavation, as it is observed in situ (Armand et al. (2014)). A $40 \times 41$ (1640) elements mesh has been used, with a fine refinement of square elements from radius 2.6 to $10.4 \mathrm{~m}$. The mesh outside $10.4 \mathrm{~m}$ is coarser, as main deformation and failure during excavation are expected only close to the gallery wall.

Regularization of the displacement field is obtained through the local second gradient model, for which parameter $D=40 \mathrm{kN}$ is chosen. Mesh sensitivity was studied on one of the microstructures under consideration, by using different mesh densities with identical model parameters and boundary conditions. Figure 13 shows the deformed meshes at the end of the computation $(\lambda=1.0)$. Comparison of the pattern of strain localization as a response to the excavation demonstrates that the parameter is large enough to regularize the displacement field for meshes with more than 30 elements per $90^{\circ}$, since the deformation is identical for finer meshes (see Figure 13).

In a second series of simulations, fluid flow is introduced at both scales to simulate the gallery excavation in 


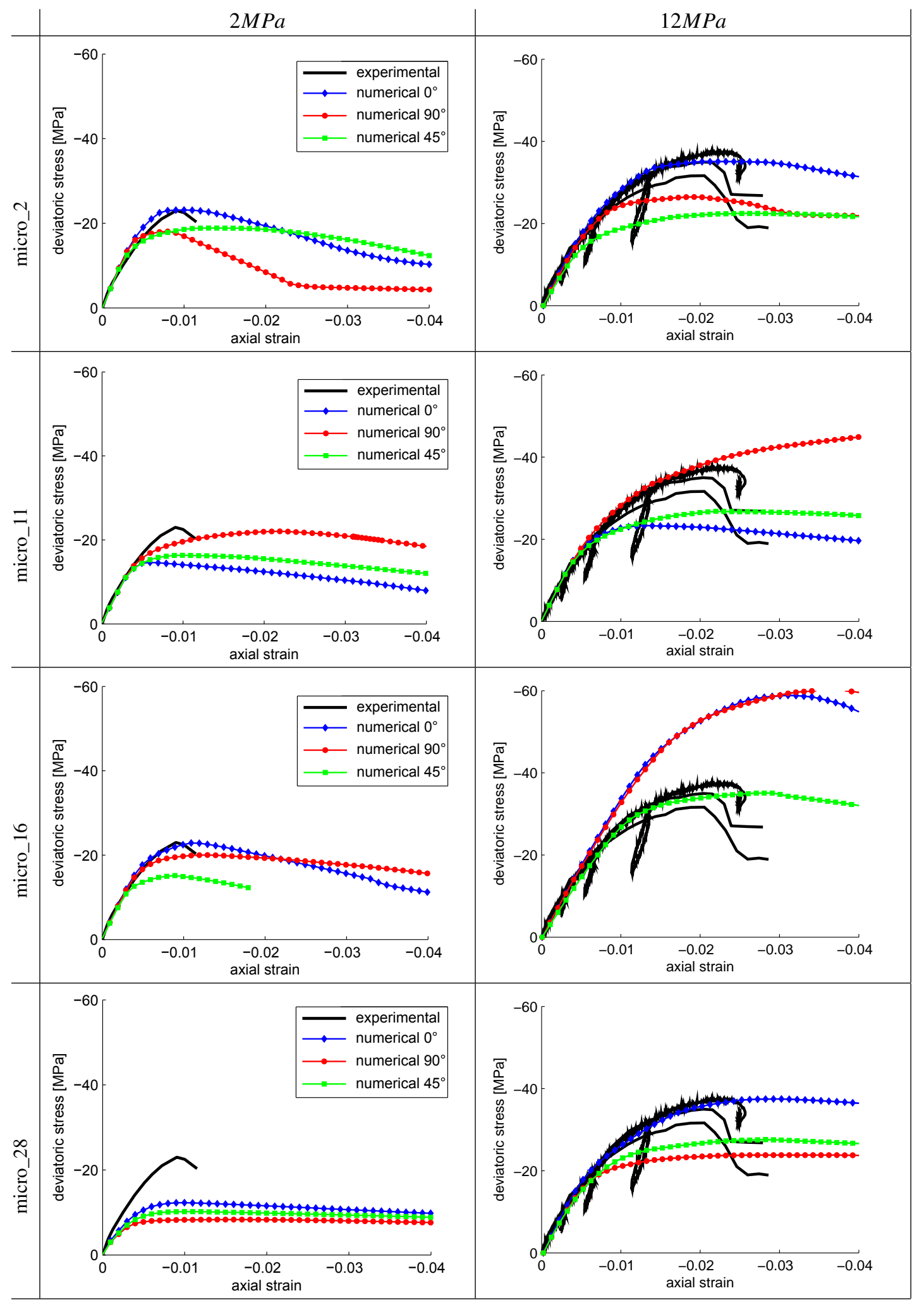

Figure 10: Results of the model calibration using biaxial compression tests at $2 \mathrm{MPa}$ and $12 \mathrm{MPa}$ lateral confining stess at different REV orientations $\theta^{R E V}$. Comparison between numerical simulation and experimental measurements. 


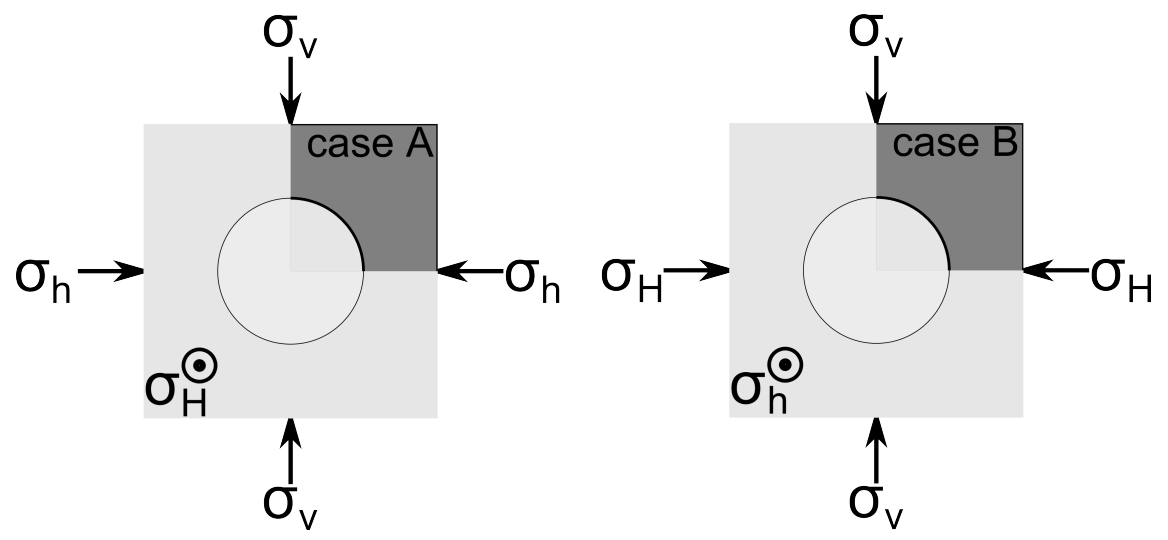

Figure 11: Orientations of galleries for case $A$ and case $B$ with respect to principal stress states.

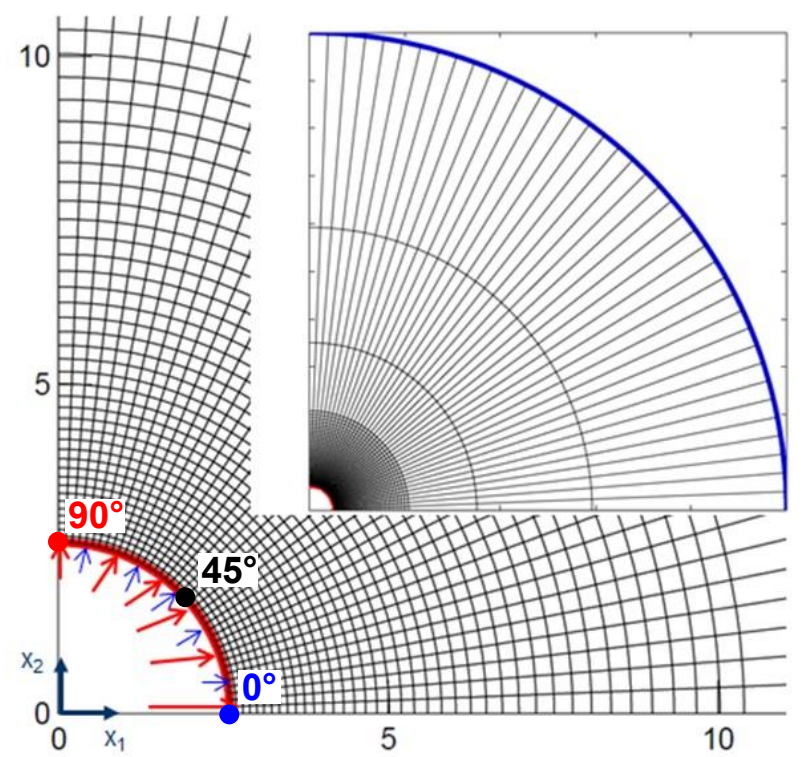

Figure 12: Mesh of the quarter hollow cylinder with a fine refinement from 2.6 to $10 \mathrm{~m}$ radius and coarse mesh between 10 and $50 \mathrm{~m}$. The red arrows represent the traction applied internally on the gallery wall and the blue arrows the pore pressure. Three points of interest for the analysis of gallery wall convergence are indicated at $0^{\circ}$ (horizontal), $45^{\circ}$ (vertical) and $90^{\circ}$ (diagonal). 

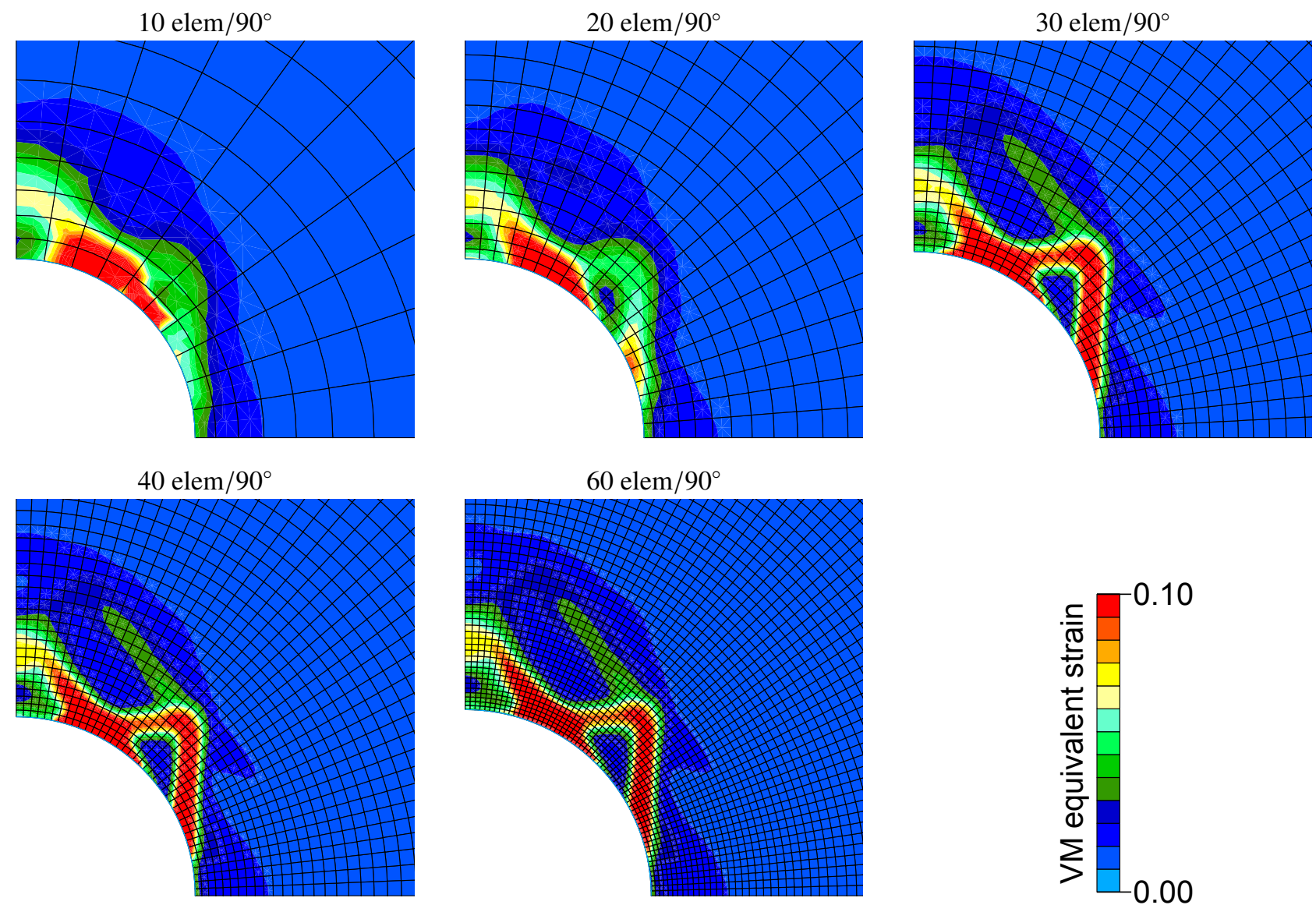

Figure 13: Strain localization patterns for case B and microstructure micro_28 with different mesh densities. Colormap is truncated at $\varepsilon_{V M}=0.10$. 


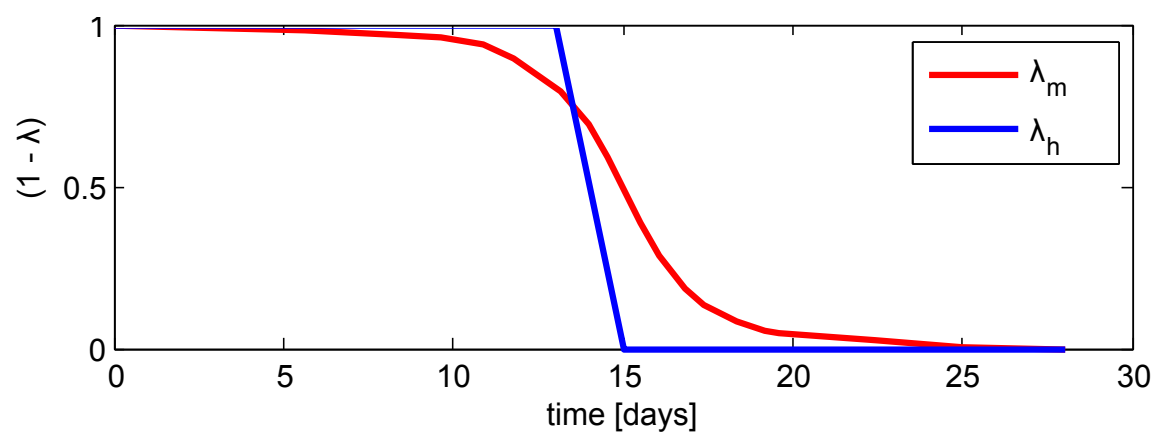

Figure 14: Time evolution of the dimensionless loading parameter $\lambda$, for the gallery wall tractions (red) and pore pressure (blue).

a water saturated argillite. The gallery is considered here parallel to the direction of minor principal stress (case B). The zone of square elements is extended to a radius of $20 \mathrm{~m}$ and the full domain is extended to $200 \mathrm{~m}$ with a very coarse mesh. Far field boundary conditions have been replaced by zero displacement conditions, for technical reasons. The initial pore pressure field is homogeneous and equal to $4.7 \mathrm{MPa}$. The pore pressure at the gallery wall progressively decreases to ambient pressure during excavation. The hydromechanical coupling introduces a time-dependency into the modelling framework due to the kinetics of pore pressure dissipation. Therefore, the time evolution of the dimensionless loading parameter $\lambda$, representing the progressive gallery excavation, has to be specified carefully to be realistic. The input of the benchmark for $\lambda$ is represented in Figure 14.

The normalized convergence criteria is fixed to $10^{-3}$ for the displacement residual and $10^{-5}$ for the force residual.

\section{Numerical modelling with a dry material}

\subsection{Isotropy of far-field stress (case A)}

The initial boundary conditions of the problem are isotropic. The solutions are shown in Figure 15 in terms of Von Mises equivalent strain rate, for the four microstructures and at three different steps of the loading ( $\lambda$ equal to $0.3,0.95$ and 1). The solutions at the beginning of loading present a rotational symmetry, which is a consequence of quasi isotropy of the model for small strains. Indeed, the macro response of the model in the elastic regime stems essentially from the (isotropic) elastic behaviour of grains at the small scale, and interfaces between grains have a marginal impact here. The macro response becomes anisotropic as soon as the constitutive relations at interfaces of the REV exceed the elastic limit. Then, the damaged interfaces exhibit some preferential orientations, depending on the stress state and loading history. From this point on, the material microstructure introduces anisotropy at the macro scale.

A strain localization can be observed as soon as $\lambda=0.95$ for the four microstructures. The strain localization pattern appears more accomplished at $\lambda=1$ where the bands are more distinct, except for micro_11 for which the bands are already formed at $\lambda=0.95$. The patterns of localization differ between microstructures. Although non uniqueness of localized solutions for excavation problems has been observed, even in case of anisotropic models (Marinelli et al., 2015), one can presuppose that the spatial position of initiation of the bands is directly influenced by the anisotropy of the model. For micro_2, the band initiates on the top of the gallery. For micro_11, the bands initiates in the sub-horizontal sector of the gallery wall. This corresponds to the zone where the orthoradial stress (major stress) is oriented in the direction of minimum strength of the microstructure (orientation $0^{\circ}$ in Figure 9) while for micro_28 and micro_16, the bands initiate in the intermediate sector between horizontal and vertical, which correspond to orientations where the orthoradial stress is aligned with some strength minimums. In most of the case, bands are conjugated which produced a gallery wall scaling when conjugated bands coalesce. The position of localization bands around the gallery wall influences the profile of gallery convergence. Gallery convergence is here defined as the radial displacement of the gallery wall towards the gallery centre, both in dry and saturated conditions. The horizontal convergence is faster in case of subhorizontal strain localization (micro_11) than the vertical convergence (Figure 16), 


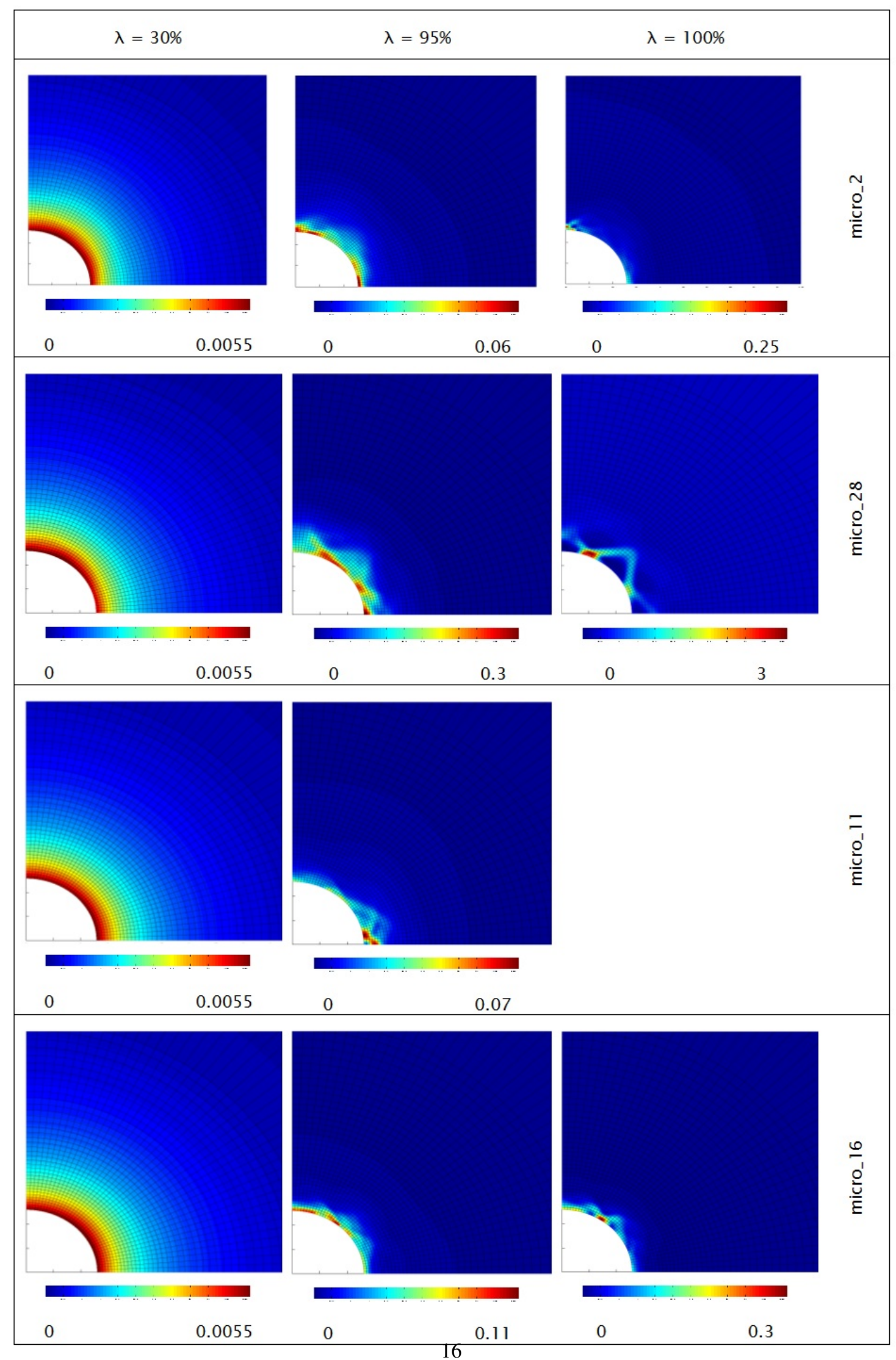

Figure 15: Von Mises equivalent strain rate fields (zoom on the gallery up to radius $10 \mathrm{~m}$ ), for the four microstructures and at three loading steps, with a dry material in case A. 

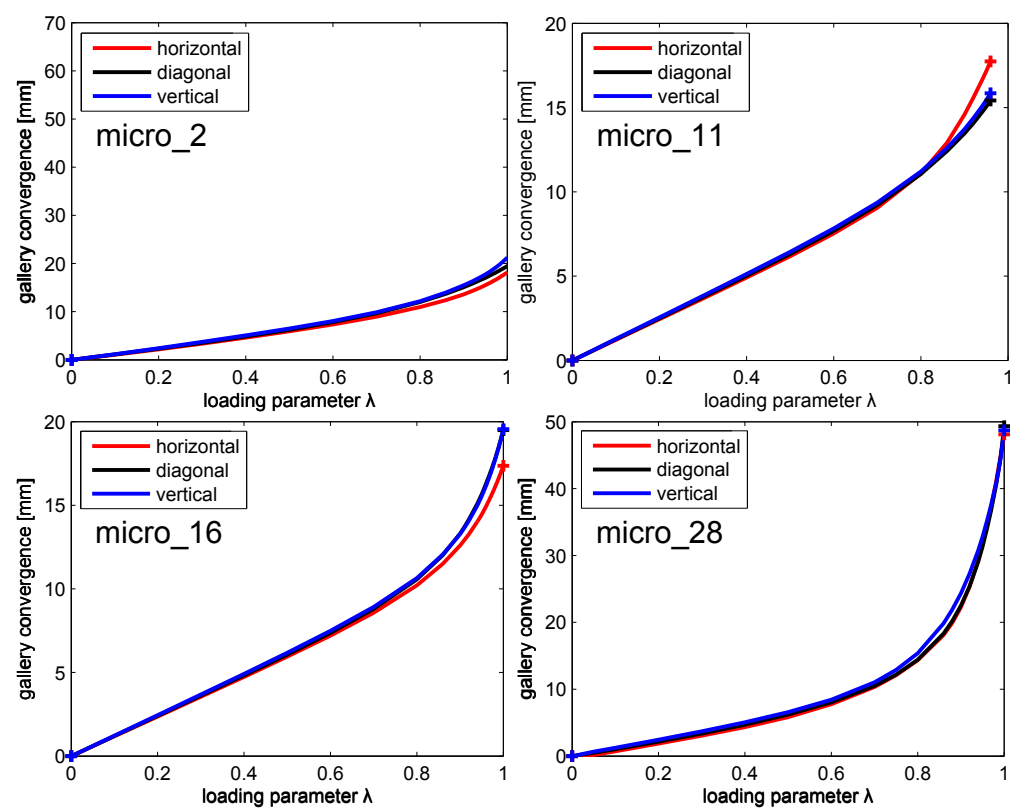

Figure 16: Gallery wall convergence along the horizontal, vertical and diagonal axis for the four microstructures, in case A.

while the tendency is reversed in case of micro_16. The rate of convergence in horizontal, vertical and $45^{\circ}$-oriented direction are equal in case of micro_28 because of symmetries of the strain localization pattern.

\subsection{Anisotropy of far-field stress (case B)}

The initial horizontal stress component is higher than the vertical stress component. Anisotropy of the solution is observed from the beginning of loading (Figure 17) by a higher deviatoric strain rate on the top of the gallery wall than on the right. In terms of localization pattern, there is a strong similarity with respect to solutions obtained with the previous boundary conditions where the far field stress is isotropic (case A). The zones around the gallery wall where the shear band initiates are more or less the same in both cases, for the four selected microstructures. The localization pattern seems influenced more by the material anisotropy than by the anisotropy of the initial stress state. The main difference is the initiation threshold of shear bands. Indeed, patterns in case B are more accomplished than in case $\mathrm{A}$ for $\lambda=0.95$. The position of shear band around the gallery wall has a strong effect on the profile of gallery convergence. When localization initiates on the top of the gallery (micro_2), the convergence of the top of the gallery accelerate significantly (Figure 18) with respect to the other directions.

\section{Numerical modelling with a water saturated material}

The effect of the hydromechanical coupling in the doublescale model on the behaviour of the EDZ is investigated. As the coupled simulation is time dependent, the boundary conditions are defined according to Figure 14, where hydraulic and mechanical boundary conditions at the gallery wall are controlled through the dimensionless loading multipliers $\lambda_{h}$ and $\lambda_{m}$ respectively:

$$
\begin{aligned}
& \vec{\tau}_{\text {gallery }}=\left(\vec{\tau}_{0}-\vec{\tau}_{\text {shield }}\right)\left(1-\lambda_{m}\right)+\vec{\tau}_{\text {shield }} \\
& p_{\text {gallery }}=p_{0}\left(1-\lambda_{h}\right)
\end{aligned}
$$

Fluid mass flux across the horizontal and vertical axes is constrained and fluid pressure is fixed to initial conditions at the farfield domain boundary (at $200 \mathrm{~m}$ from the gallery). Initial total stress state is anisotropic according to case B with total stress components $\sigma_{H}=16.1 \mathrm{MPa}$ and $\sigma_{v}=12.7 \mathrm{MPa}$. Initial pore pressure is $\mathrm{p}^{0}=4.7 \mathrm{MPa}$ and the 


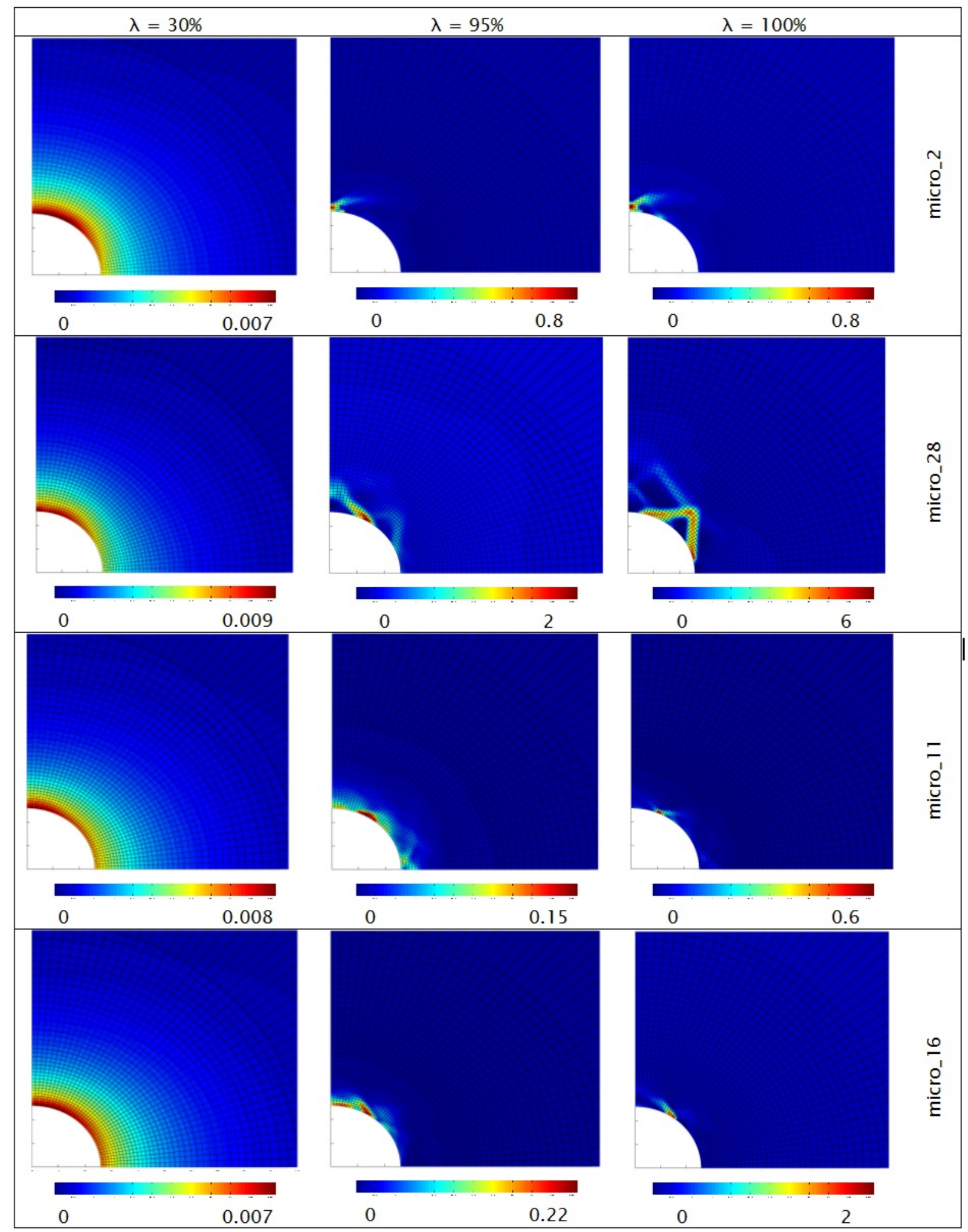

Figure 17: Von Mises equivalent strain rate fields (zoom on the gallery up to radius $10 \mathrm{~m}$ ), for the four microstructures and at three loading steps, with a dry material in case B. 


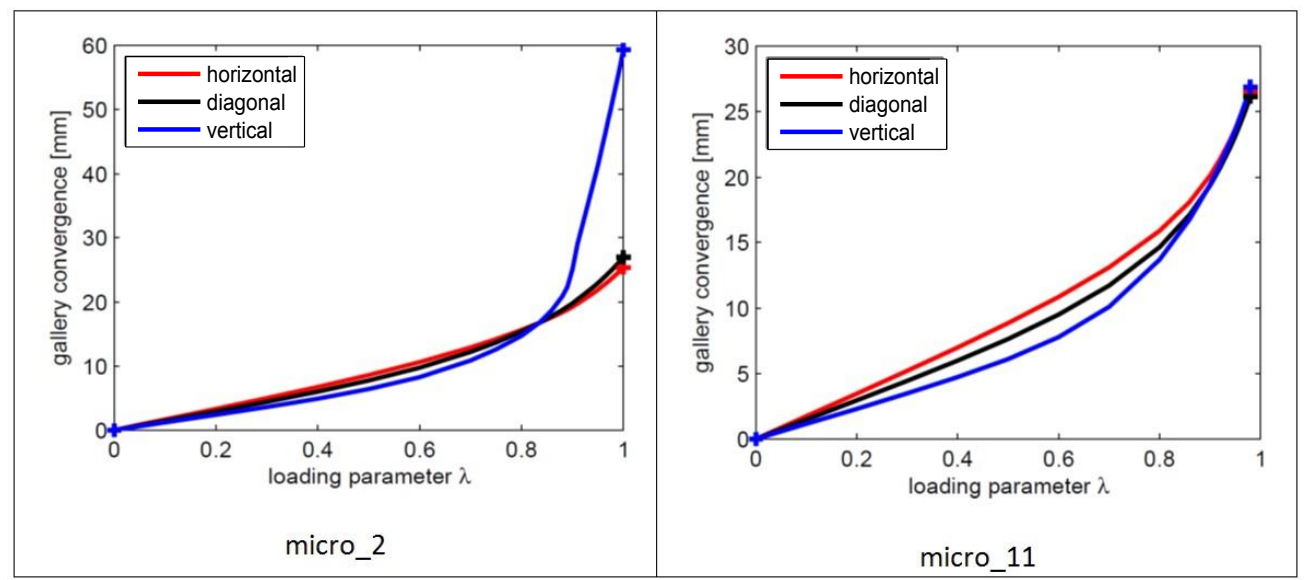

Figure 18: Gallery wall convergence in horizontal direction $\left(0^{\circ}\right), 45^{\circ}$-direction $\left(45^{\circ}\right)$ and vertical direction $\left(90^{\circ}\right)$, for micro_2 and micro_11, in case B.

system is in equilibrium at time $T=0$. Only microstructure micro_28 is used for the simulation under saturated conditions.

Compared to the dry simulations, which are time-independent and reach an equilibrated state at the point of complete excavation ( $\mathrm{T}=28$ days or $\lambda=1$ ), the simulation of an excavation in fully saturated material has a time dependent response, which is partly controlled by the decrease of the traction on the gallery wall and partly by the dissipation of the pore pressure differences, induced by this unloading. Figure 19 shows the deformation and pore pressure fields at different stages during and after excavation. VM strain (left) is used to indicate the state of total deformation whereas VM strain rate (centre) indicates the current activity of the deformation process.

As a response to the excavation, only the zones close to the gallery wall show deformation activity during the excavation (upto 28 days). Further away from the gallery wall negative pore pressures prevent most of the deformation. The negative pore pressures tend to dissipate, allowing further deformation away from the gallery wall. With the negative pore pressures sufficiently dissipated, the pattern of strain localization bands that forms is similar to the pattern observed for the dry simulation. The hydromechanical coupling partly prevents deformation during and shortly after the excavation and thereby has a delaying effect on the gallery convergence. Note that the contour plots in Figure 19 have unequal color maps as to highlight the spatial variation of the level of strain and pore pressure. A quantitative comparison between the different stages can be done by means of Figures 20, 21 and 22.

Figure 20 shows the gallery wall convergence with time, where $\mathrm{T}=28$ days corresponds to complete excavation. During the period of active excavation, the gallery wall convergence is mainly controlled by the decrease of mechanical support as the response is practically undrained. After excavation, the response is fully controlled by pore pressure dissipation. Figure 21 shows the pore pressure evolution for points at $1 \mathrm{~m}$ and $2 \mathrm{~m}$ from the gallery wall along the horizontal, vertical and diagonal axes. The minimum pore pressure is reached later at points further away from the gallery wall. This confirms the delaying effect of the hydromechanical coupling and the dissipation of the peak of underpressure after excavation. Figure 22 contains the pore pressure profiles along the horizontal and vertical axes at different stages after excavation and shows the dissipation of the underpressure peak towards an equilibrium state. The pore pressure evolution is a coupled effect of pore volume change due to deformation and fluid mass transport from the far field towards the gallery wall. The strong underpressure as a response to deformation indicates a dilatant behaviour of the material. This is in line with the micromechanical mode of deformation, in which relative displacement of grains can only take place when interfaces open with respect to the undeformed configuration. This results in the increasing pore volume with deformation.

The negative pore pressures as a reaction to the dilatant behaviour of the model is maintained throughout the simulation, because only saturated conditions are considered. It can be argued that in reality, these levels of negative pore pressure can only be obtained by suction under unsaturated conditions. These undrained conditions would then play an important role in the fluid mass balance of the problem. This has important implications for the applicability of the model, as will be discussed in the next section 

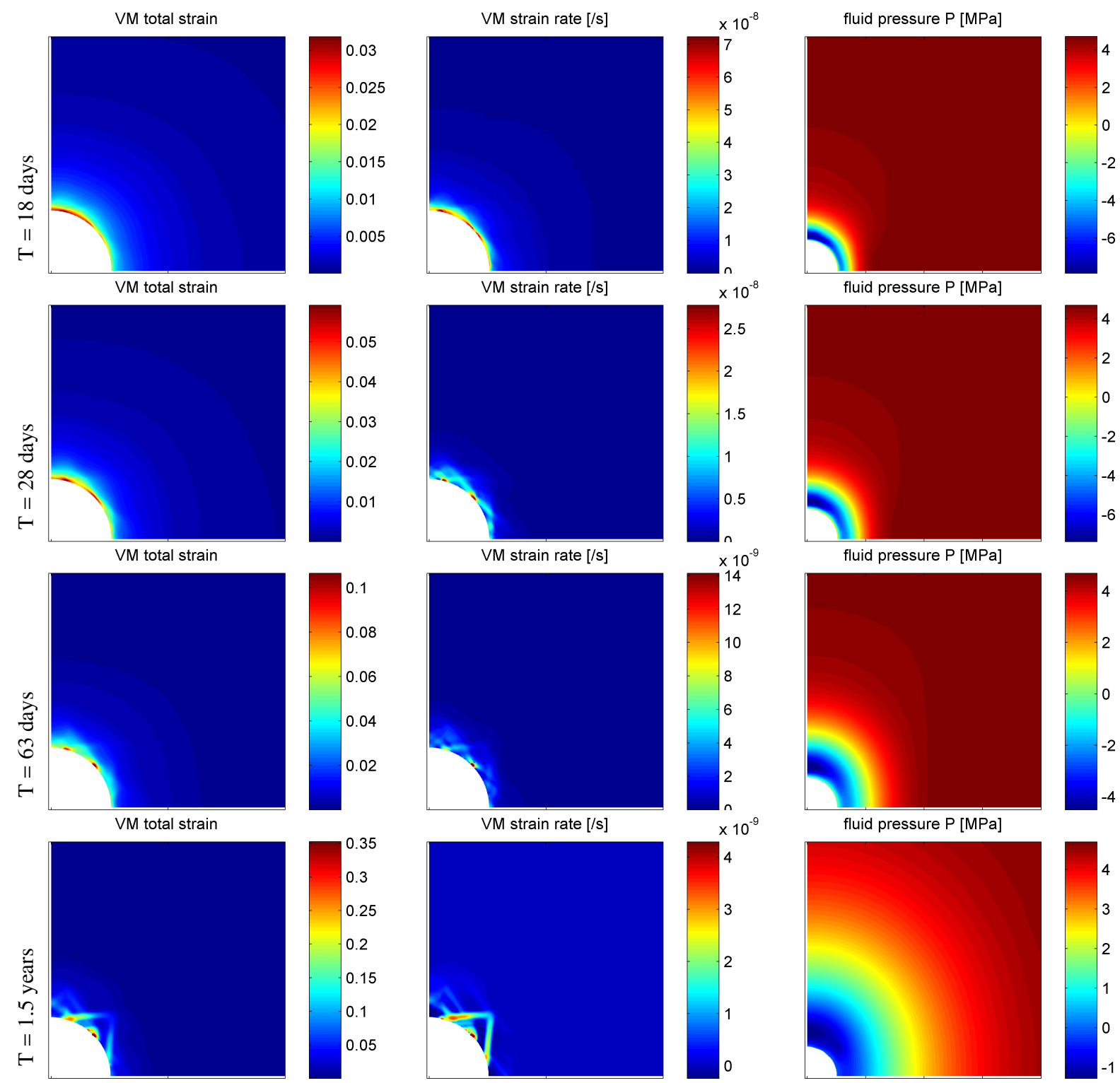

Figure 19: Deformations and pore pressure fields around the excavated gallery at different instances for the simulation of case B with hydromechanical coupling. 


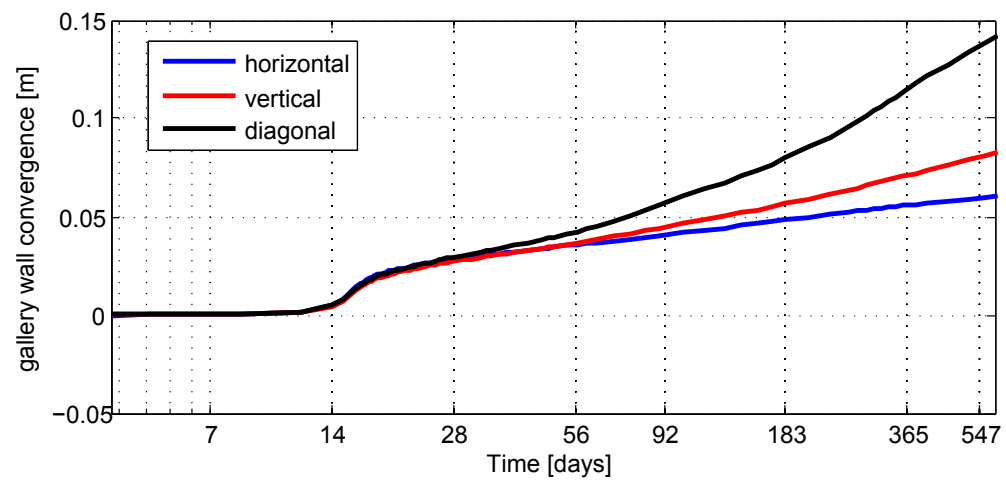

Figure 20: Gallery convergence towards the gallery center along the horizontal, vertical and diagonal axes, demonstrating the deformation delay due to the hydromechanical coupling.

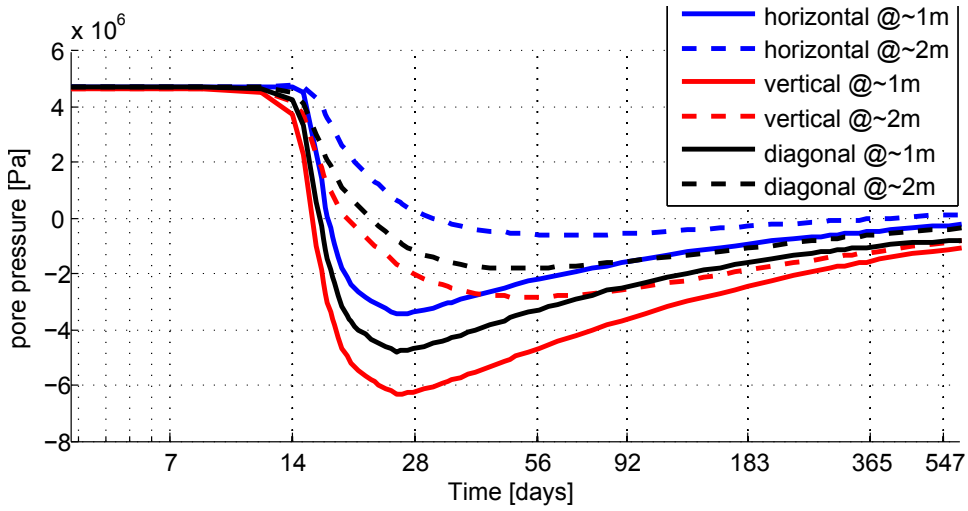

Figure 21: Pore pressure evolution during and after excavation at $1 \mathrm{~m}$ and $2 \mathrm{~m}$ distance of the gallery wall, showing the delayed effect due to pore pressure dissipation.

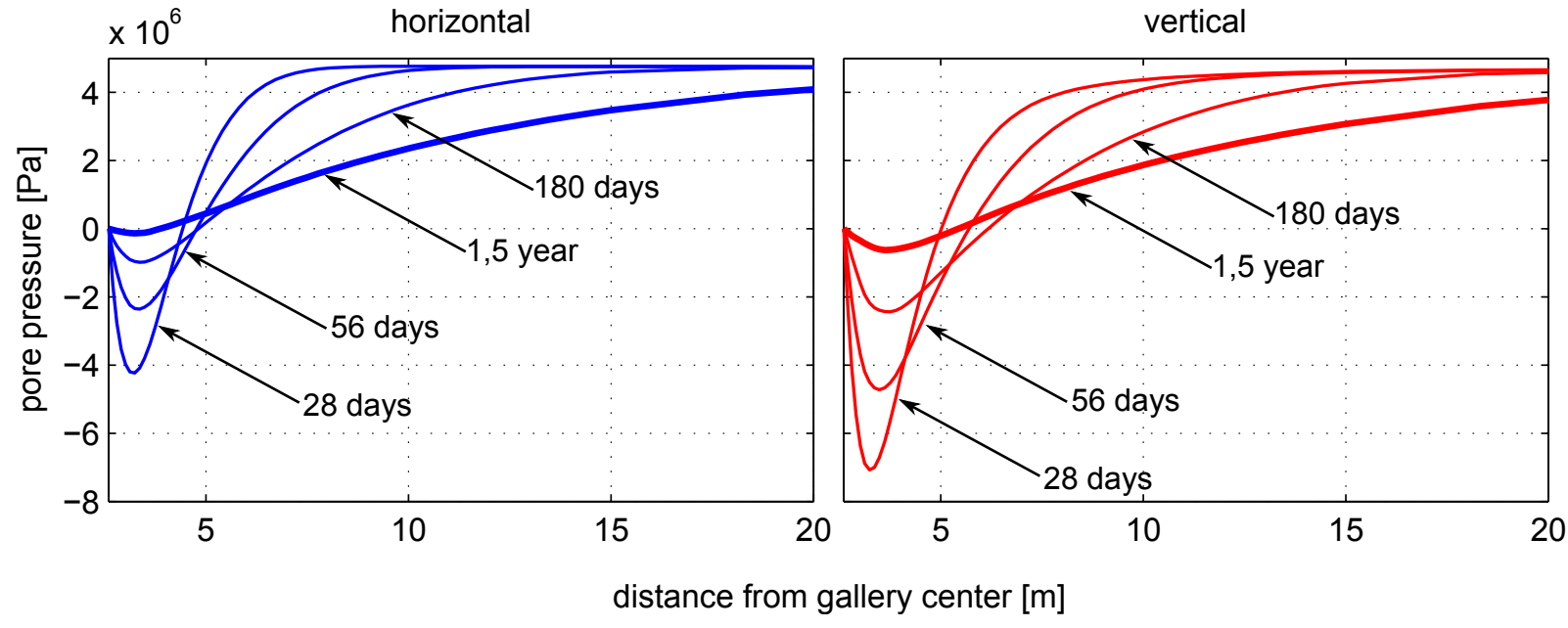

Figure 22: Pore pressure profiles at different stages of pore pressure dissipation along the horizontal and vertical axes. 


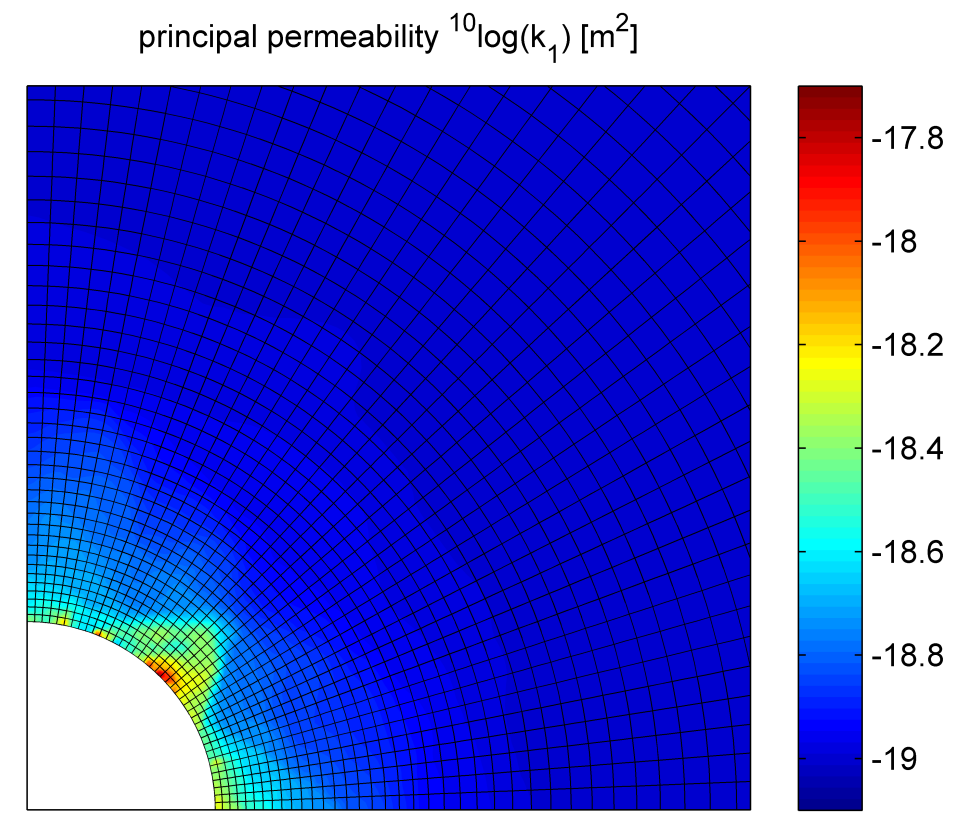

Figure 23: Principal component of permeability tensor at $\mathrm{T}=1.5$ year

The rate of dissipation is strongly dependent on the permeability of the material. Through the definition of a minimum interface opening, the permeability of undeformed material is modelled at $k_{i i} \approx 5 \times 10^{-20} \mathrm{~m}^{2}$. As an effect of the deformation of the microstructure, an increase of the permeability can be observed in the excavation damaged zone. Figure 23 shows the principal component of the permeability tensor at $T=1.5$ year. Compared with the pattern of deformation observed in Figure 19, it is clear that enhancement of permeability shows correspondence with the pattern of localization of deformation.

\section{Discussion}

The application of the multiscale computational framework on the engineering-scale benchmark problem has demonstrated the possibility to use $\mathrm{FE}^{2}$ methods for hydromechanical couplings on engineering scale. Computations were performed on a regular desktop machine without relying on parallel computation. With a computation time between 4 and 10 minutes per macroscale iteration, computations of the dry doublescale simulations took 1 to 2 days to perform, depending on the quality of numerical convergence of loading steps and the need for reduction of the loading step size to obtain this convergence. For the case of hydromechanical coupling, many more time steps were needed first of all for a proper time integration of the transient problem and secondly to obtain good convergence of the macroscale Newton-Raphson iterative scheme. This has led to a total computation time of approximately 2 weeks for the simulation of hydromechanical coupling. It should be noted that the microscale computations on individual REVs take most (about 95\%) of the computation time. As these computations, four for each macroscale element, are independent, the method is highly suitable for parallel computing, allowing a speedup of orders of magnitude when enough CPUs are available.

Anisotropy in the macroscale constitutive relations results directly from the microscale computations, due to the heterogeneity of the microstructure and the spatial distribution of the interfaces (grain to grain contacts and fluid channels). Influenced by the stress state and the history of the loading path with respect to the REV orientation, preferential crack damage appears. It induces an anisotropy of the stiffness moduli at the macroscale that influences the localization pattern. A better control of the anisotropy deserves further developments. This would require larger REVs with more grains and interfaces. The low influence of the anisotropy of the in situ stress state observed on the localization pattern simulations, that does not agree the in situ observations (Armand et al., 2016), is probably a 
matter of model anisotropy. Another point to address is the loss of periodicity of the REV in the softening regime. When periodicity of the microstructural deformation is lost, so is the objectivity of the response with respect to the boundary conditions of the REV (Bilbie et al., 2008). In this case, the REV dimensions and orientation become an inherent part of the material response, introducing a length scale relative to the REV size. Although several enhancements of the REV boundary conditions were proposed in literature (Coenen et al., 2011; Toro et al., 2014) a fully objective enhancement capable of consistently taking into account fluid mass transport is not likely to be readily available. Meanwhile, the effect of the boundary conditions is accepted as part of the material response and first order computational homogenization is used for the scale transition.

Assumption of symmetries of the BVP that allows to reduce the full gallery problem to a quarter gallery problem is not valid in case of arbitrary anisotropy of the model. Moreover, loss of uniqueness of the localized solutions in the excavation problem was observed with both isotropic and anisotropic elasto-plastic models (Marinelli et al., 2015). Several solutions exist and the convergence toward a solution is particularly sensitive to numerical details (time step, convergence criteria, etc...). These solutions generally not satisfy the spatial symmetries assumed when modelling only a quarter of the problem. Further developments would be needed to investigate these aspects with the $\mathrm{FE}^{2}$ model.

The obtained evolution of the permeability is strongly influenced by the modelling of the microstructure in a 2D model. This restriction prevents taking into account the 3 -dimensional nature of fluid percolation paths. The confined stress state prevents the development of continuous flow paths of opened interfaces in $2 \mathrm{D}$, as the assembly of grains has to remain in contact. In consequence, the permeability change is underestimated, while the dilatancy was probably overestimated in the model. Indeed, the local increase in permeability of several orders of magnitude as observed by Armand et al. $(2013,2014)$ was not obtained in the numerical simulations. Although the extension to a 3-dimensional $\mathrm{REV}$ is reported not to lead directly to an increase of several orders of magnitude (Massart and Selvadurai, 2012), it can be considered as a significant step towards a better physical representation of fluid percolation at the microscale.

Due to the restricted complexity of both the microstructure taken into account and the first-version model, a fair quantitative comparison with in-situ observations is not possible. Although it was demonstrated that several aspects of the material behaviour can be reproduced with reasonable accuracy, others can not be obtained with the current model. Plastic deformation can not be taken into account in the current version of the microscale model, although an extension of the model would not require any modifications of the multiscale framework. This is not the case for time-dependent behaviour, which would not fit into the current framework.

The model was formulated for saturated conditions. Without restrictions on the minimum fluid pressure, this can give unrealistic pore pressure values as observed in the final simulation. An extension to unsaturated or three-phase (solid-fluid-gas) formulation would require a significant extension of the multiscale framework.

\section{Conclusion}

A multiscale modelling approach for hydromechanical coupling by means of the $\mathrm{FE}^{2}$ method in the framework of computational homogenization was applied to the simulations of gallery excavation in the "Transverse action" benchmark. A first-version micromechanical model for solid-fluid interaction was adopted to characterize coupled behaviour at the microstructure scale. It can be concluded that the model, by adipting the first-version model without further conceptual development, is capable of capturing the global characteristic of strain localization in the excavation damaged zone. The difference in microstructure was demonstrated to have an important effect on the anisotropy of the macroscale material behaviour and thereby on the initiation and progression of strain localization around the gallery wall. This material anisotropy seems to have significant effects compared to the in situ stress anisotropy. Indeed, a qualitative comparison of localization patterns between isotropic and anisotropic BVP shows the determining influence of the material anisotropy.

\section{Acknowledgements}

The first author thanks ANDRA (French national agency for nuclear wastes storage) for financial support. The 3SR laboratory is part of the LabEx Tec 21 (Investissements d'Avenir - grant agreement $\mathrm{n}^{o}$ ANR-11-LABX-0030). 


\section{Bibliography}

Andra, 2005. Dossier 2005 argile : Synthèse : Evolution phénoménologique du stockage géologique. Tech. rep., ANDRA, France.

Andra, 2013. Action Modèles transverse au GL Géomécanique et au Programme de simulation de l'Andra (UPS4) - Phase 3: modélisation des ouvrages. CG.RP.AMFS.13.0031. Tech. rep., ANDRA, France.

Armand, G., Leveau, F., Nussbaum, C., de la Vaissiere, R., Noiret, A., Jaeggi, D., Landrein, P., Righini, C., 2014. Geometry and properties of the excavation-induced fractures at the meuse/haute-marne url drifts. Rock Mechanics and Rock Engineering 47 (1), $21-41$.

Armand, G., Noiret, A., Zghondi, J., Seyedi, D., 2013. Short- and long-term behaviors of drifts in the Callovo-Oxfordian claystone at the Meuse/Haute-Marne Underground Research Laboratory. Journal of Rock Mechanics and Geotechnical Engineering 5 (3), 221 - 230.

Armand et al., G., 2016. Fundamental aspects of the hydromechanical behavior of the callovo-oxfordian claystone - from experimental investigations toward a modeling perspective. Computers and Geotechnics - submitted, same issue.

Bésuelle, P., Chambon, R., Collin, F., 2006. Switching deformation modes in post-localization solutions with a quasibrittle material. J. Mech. Mat. Str. $1,1115-1134$

Bilbie, G., Dascalu, C., Chambon, R., Caillerie, D., 2008. Micro-fracture instabilities in granular solids. Acta Geotechnica 3 (1), $25-35$.

Biot, M., 1941. General theory of three-dimensional consolidation. Journal of Applied Physics 12 (2), $155-164$.

Chambon, R., Caillerie, D., 1999. Existence and uniqueness theorems for boundary value problems involving incrementally non linear models. International Journal of Solids and Structures 36 (33), $5089-5099$.

Chambon, R., Caillerie, D., Matsuchima, T., 2001. Plastic continuum with microstructure, local second gradient theories for geomaterials: localization studies. International Journal of Solids and Structures 38 (46-47), $8503-8527$.

Charlier, R., 1987. Approche unifiée de quelques problèmes non linéaires de mécanique des milieux continus par éléments finis. Ph.D. thesis, Ph. D. Thesis, Dept. MSM Université de Liège.

Coenen, E., Kouznetsova, V., Geers, M., 2011. Novel boundary conditions for strain localization analyses in microstructural volume elements. Int. J. for Num. Meth. in Eng. 90, $1-21$.

Collin, F., Chambon, R., Charlier, R., 2006. A finite element method for poro mechanical modelling of geotechnical problems using local second gradient models. International Journal for Numerical Methods in Engineering 65 (11), 1749-1772.

Feyel, F., Chaboche, J.-L., 2000. FE ${ }^{2}$ multiscale approach for modelling the elastoviscoplastic behaviour of long fibre sic/ti composite materials. Computer Methods in Applied Mechanics and Engineering 183 (3-4), 309 - 330.

Frey, J., Dascalu, C., Chambon, R., 2012. A two-scale poromechanical model for cohesive rocks. Acta Geotechnica 7, 1-18.

Geers, M., Kouznetsova, V., Brekelmans, W., 2010. Multi-scale computational homogenization: Trends and challenges. Journal of Computational and Applied Mathematics 234 (7), 2175 - 2182.

Germain, P., 1973. La méthode des puissances virtuelles en mécanique des milieux continus. J. Mécanique 12, $235-274$.

Kouznetsova, V., Brekelmans, W. A. M., Baaijens, F. P. T., 2001. An approach to micro-macro modeling of heterogeneous materials. Computational Mechanics 27 (1), 37-48.

Marinelli, F., Sieffert, Y., Chambon, R., 2015. Hydromechanical modeling of an initial boundary value problem: Studies of non-uniqueness with a second gradient continuum. International Journal of Solids and Structures 54, $238-257$.

Marinelli, F., van den Eijnden, A., Sieffert, Y., Chambon, R., Collin, F., 2016. Modeling of granular solids with computational homogenization: Comparison with biot's theory. Finite Elements in Analysis and Design 119, 45 - 62. URL http://www.sciencedirect.com/science/article/pii/S0168874X16300725

Massart, T., Selvadurai, A., 2012. Stress-induced permeability evolution in a quasi-brittle geomaterial. Journal of Geophysical Research 117, 1-15.

Matsushima, T., Chambon, R., Caillerie, D., 2002. Large strain finite element analysis of a local second gradient model: application to localization. Int. J. for Num. Meth. in Eng. 54, 499-521.

Mindlin, R., 1964. Micro-structure in linear elasticity. Archive for Rational Mechnaics and Analysis 16, 51-78.

Özdemir, I., Brekelmans, W. A. M., Geers, M. G. D., 2008. Computational homogenization for heat conduction in heterogeneous solids. International Journal for Numerical Methods in Engineering 73 (2), 185-204.

Robinet, J.-C., Sardini, P., Coelho, D., Parneix, J.-C., Prêt, D., Sammartino, S., Boller, E., Altmann, S., 2012. Effects of mineral distribution at mesoscopic scale on solute diffusion in a clay-rich rock: Example of the callovo-oxfordian mudstone (Bure, France). Water Resources Research $48(5)$.

Seyedi, D., Armand, G., Noiret, A., 2016. 'transverse action' - a model benchmark exercise for numerical analysis of the Callovo-Oxfordian claystone hydromechanical response to excavation operations. Computers and Geotechnics - submitted, same issue.

Terada, K., Kikuchi, N., 1995. Nonlinear homogenization method for practical applications. ASME applied mechanics division-publications-AMD, $1-16$.

Toro, S., Sï£;nchez, P., Huespe, A., Giusti, S., Blanco, P., Feijį;o, R., 2014. A two-scale failure model for heterogeneous materials: numerical implementation based on the finite element method. International Journal for Numerical Methods in Engineering 97 (5), $313-351$.

van den Eijnden, A., Bï£;suelle, P., Collin, F., Chambon, R., 2016. A FE ${ }^{2}$ modelling approach to hydromechanical coupling in cracking-induced localization problems. (to be) accepted under minor revisions for publication in International Journal of Solids and Structures.

van den Eijnden, B., 2015. Multiscale modelling of the hydromechanical behaviour of argillaceous rocks. Ph.D. thesis, Universitï $;$ de Grenoble. 